\title{
Neurexin and Frizzled signaling intercept axonal-transport at microtubule minus-ends to control synapse formation
}

Santiago Balseiro-Gómez ${ }^{1}$, Yang Yue ${ }^{2}$, Lin Shao ${ }^{1}$, Selim Çetinkaya ${ }^{1}$, Caroline Kuzoian ${ }^{1}$, Kristen J Verhey ${ }^{2}$, Shaul Yogev ${ }^{*}$

${ }^{1}$ Department of Neuroscience, Yale School of Medicine, 295 Congress Ave, New Haven, CT 06510

${ }^{2}$ Department of Cell and Developmental Biology, University of Michigan, Ann Arbor, MI 48109

* Corresponding author and lead contact

Contact: shaul.yogev@yale.edu (203) 785-7772 


\section{$\underline{\text { Abstract }}$}

Precise synaptic connectivity defines neuronal circuits. Synapse formation is locally determined by transmembrane proteins, yet synaptic material is synthesized remotely and undergoes processive transport in axons. How local synaptogenic signals intercept synaptic cargo in transport to promote its delivery and synapse formation is unknown. We found that control of cargo delivery from microtubule minus-ends mediates pro- and anti-synaptogenic activities of presynaptic Neurexin and Frizzled in $C$. elegans, and identified the atypical kinesin VAB-8/KIF26 as a key molecule in this process. VAB-8/KIF26 levels on synaptic microtubule minus-ends are controlled by Frizzled and Neurexin, its loss mimics neurexin mutants or Frizzled hyperactivation, and its overexpression can rescue synapse-loss in these backgrounds. VAB-8/KIF26 protects other minus-end proteins and promotes pausing of retrograde transport to allow delivery into synapses. Consistently, reducing retrograde transport rescues synapseloss in vab-8 and neurexin mutants. These results uncover an important mechanistic link between synaptogenic signaling and axonal transport.

\section{Introduction}

The proper function of complex neuronal circuits hinges on the establishment and maintenance of precisely patterned synaptic connections. Synapse position and numbers are instructed by local proand anti-synaptogenic signals from cell adhesion molecules such as Neurexin/Neuroligin and diffusible cues such as Wnts and their Frizzled (Fz) receptors (Sanes and Zipursky, 2020; Südhof, 2018; Yogev and Shen, 2014). In parallel, most synaptic proteins are synthesized in the cell-body and rely on long-range axonal transport to arrive at synapses (Guedes-Dias and Holzbaur, 2019; Maeder et al., 2014; Rizalar et al., 2021). A key question which remains poorly understood is how the activity of synaptogenic signals locally controls the delivery of cargo undergoing transport to generate the appropriate synaptic connectivity pattern.

Presynaptic Neurexins are key synaptse-organizing molecules, with variable context-dependent effects on synapse formation and maturation. Neurexin's extra-cellular domain binds a range of postsynaptic ligands to specify synapse properties (Südhof, 2017). Despite the identification of Neurexin intracellular domain binding proteins, the signaling events downstream of presynaptic neurexin are poorly understood (Biederer and Südhof, 2000; Brouwer et al., 2019; Hata et al., 1996; Owald et al., 2012). Wnts and their Fz receptors are universal signaling molecules with diverse developmental and homeostatic roles. Unlike Neurexin, which largely acts to promote synapse formation or specialization, Fz can either promote or inhibit synapse formation (He Chun-Wei et al.; Koles and Budnik, 2012; Sanes and Zipursky, 2020). Both Fz and presynaptic Neurexin can signal to the synaptic cytoskeleton, although how this promotes or inhibits synapse formation is not well understood (Biederer and Sudhof, 2001; Lüchtenborg et al., 2014; Miech et al., 2008; Muhammad et al., 2015; Sugie et al., 2015).

Synapses rely on long-range microtubule-based transport of synaptic cargo from the cell body (Kreutzberg, 1969). Electron and light-microscopy studies identified "transport packets" containing synaptic vesicle precursors (SVPs) and active zone (AZ) proteins that are delivered by fast axonal transport (Ahmari et al., 2000; Vukoja et al., 2018; Wu et al., 2013; Zhai et al., 2001). Genetic analysis identified the kinesin-3 KIF1A/UNC-104 as the key motor for long-range transport of presynaptic cargo, as cargo is retained in the cell body of kinesin-3 mutants (Hall and Hedgecock, 1991; Okada et al., 1995; Pack-Chung et al., 2007). Intuitively, it is reasonable to expect that all cargo delivery into synapses would occur by local regulation of kinesin-3 at synapses. However, live-imaging studies of en passant synapses 
clearly show that synaptic cargo is also delivered into presynaptic boutons during retrograde transport from the distal axon towards the cell body, suggesting a role for the retrograde motor dynein (Bharat et al., 2017; Wong et al., 2012). Although several synaptic mechanisms that locally promote capture of dense core vesicles (DCVs) from Kif1A/kinesin-3 at pre and post synaptic sites were identified (Bharat et al., 2017; Stucchi et al., 2018), how cargo capture during retrograde transport is regulated is unknown.

Dynamic microtubule plus-ends are abundant at synapses and were recently shown to promote SVP delivery during anterograde transport by kinesin-3, which runs to the tip of the microtubule and dissociates from the GTP cap (Guedes-Dias et al., 2018; Qu et al., 2019). Studies in vitro and in axons have shown that retrograde transport preferentially pauses at microtubule minus-ends (Soundararajan and Bullock, 2014; Tan et al., 2018; Yogev et al., 2016), raising the possibility that these ends might be sites where dynein-driven transport is regulated. However, compared to the large number of proteins identified at microtubule plus-ends, our understanding of microtubule minus-ends in neurons is rudimentary, with few proteins known to reside there (Akhmanova and Steinmetz, 2019; van de Willige et al., 2016).

Here, we found that control of synapse number and positioning by C. elegans Neurexin (NRX-1) and the Fz receptor MIG-1 converges on cargo delivery from microtubule minus-ends. We identify the atypical kinesin VAB-8/KIF26 as a microtubule minus-end resident protein that plays a critical role in this process. NRX and Fz signaling locally control the stability of VAB-8/KIF26 on a subset of synaptic microtubules, with degradation of VAB-8/KIF26 by Fz signaling antagonizing the pro-synaptogenic activity of neurexin. Local loss of VAB-8 from synaptic microtubule minus-ends results in impaired distribution of other MT minus-end proteins PTRN-1/CAMSAP and NOCA-1/Ninein, and in excessively processive retrograde transport, which occurs at the expense of cargo delivery and leads to synapse loss. Consistently, reducing dynein activity restores the pattern of presynaptic sites in vab-8/KIF26 mutants, in neurexin mutants, and in a mutant that leads to excessive Fz signaling. These results uncover cargo delivery from microtubule minus-ends as a mechanism for sculping synaptic connectivity by Neurexin and Frizzled signaling.

\section{$\underline{\text { Results }}$}

\section{The Fz receptor MIG-1 acts antagonistically to Neurexin in synapse formation}

The $C$. elegans cholinergic motor neuron DA9 resides in the preanal ganglion, from where it extends a dendrite in the ventral cord and an axon that traverses across to the dorsal cord and grows anteriorly, establishing 25 en passant presynaptic sites onto dorsal muscles at stereotypic positions (Figure 1A) (White et al., 1986). Expression of the active zone protein Clarinet-1 (CLA-1), homolog of mammalian Piccolo and Bassoon, and the SVP marker RAB-3 allow to visualize DA9 synapses at a singlecell resolution (Chen et al., 2018; Kurshan et al., 2018; Xuan et al., 2017) (Figure 1B). In DA9, the sole $C$. elegans Neurexin, $n r x-1$, is required presynaptically for at least two known functions: clustering of RAB-3 vesicles and preventing loss of proximal synaptic boutons (Kurshan et al., 2018) (Figure 1E).

We noticed that a mutant isolated in a forward genetic screen for regulators of microtubule minus-ends (see below, Figure 5), shy20, also displayed a loss of proximal synapses, highly reminiscent of $n r x-1$ (wy778) mutants (Figure 1D), an allele which deletes the $\alpha$ and $\gamma$ isoforms (For description of alleles, refer to Table S1. All alleles used in this study are strong loss of function or null alleles, unless 


\section{Figure 1}
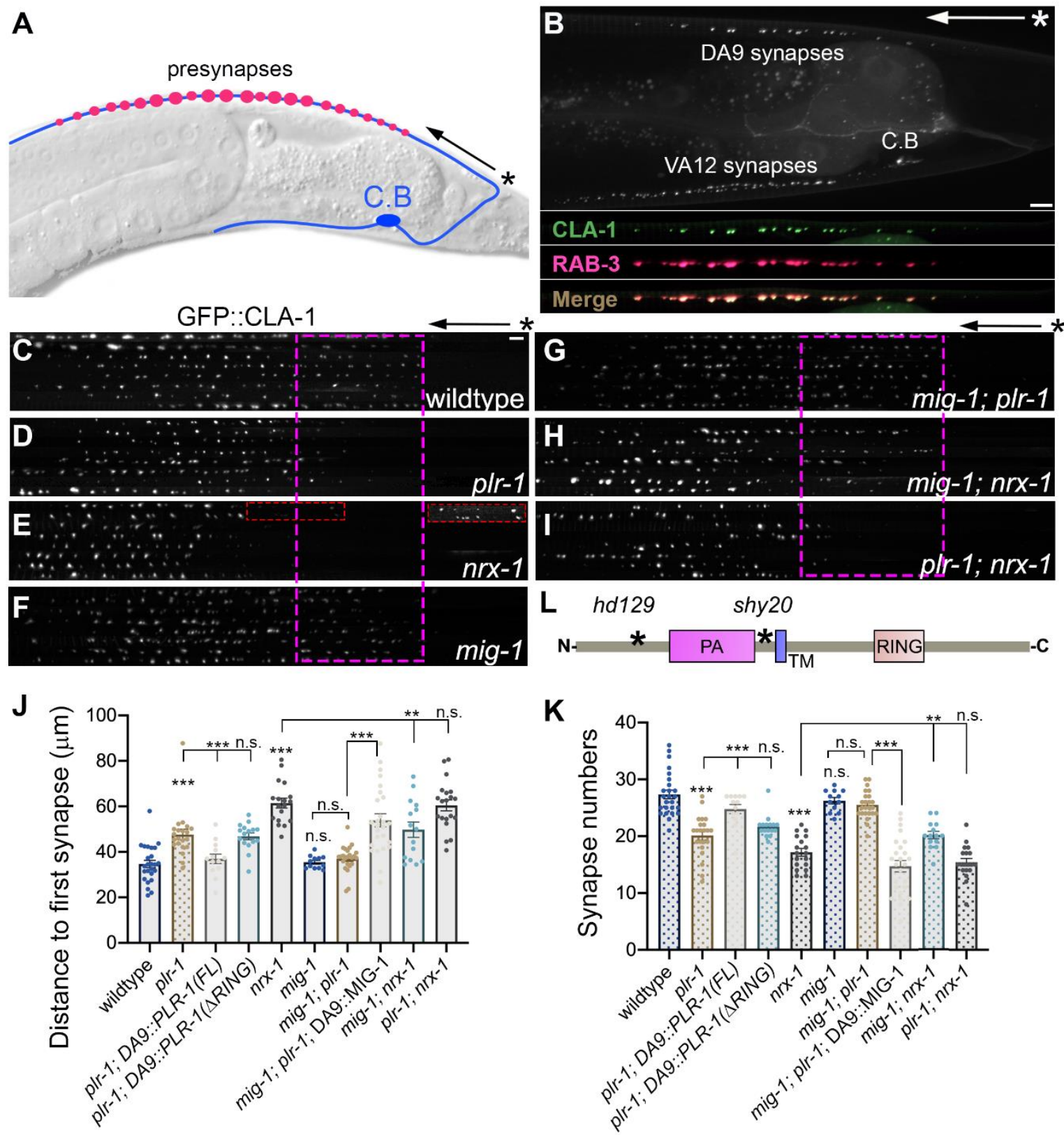

Figure 1: Fz/MIG-1 signaling acts antagonistically to Neurexin in synapse formation.

(A) Schematic of the DA9 motor neuron. 25 en passant presynapses form at stereotypic position along the axon. ${ }^{*}$ and arrow indicate the turn of the commissure and asynaptic domain in all figures. (B) wildtype adult expressing the active zone marker GFP::CLA-1 and the synaptic vesicle marker tdTomato::RAB-3 under the Pmig-13 promoter. VA12 synapses on the ventral side are also labelled by the transgene. Bottom panels show a straightened section of the axon beginning at the turn of the commissure $\left(^{*}\right)$. C.B, DA9 cell body. Scale bar $5 \mu \mathrm{m}$. (C-I) mig-1 partially suppresses $n r x-1$ mutants. Confocal images of wildtype $(C)$ and indicated mutant genotypes expressing the synaptic marker GFP::CLA-1. Eight axons per genotype were straightened and aligned from the turn of the commissure $(*)$. Magenta square highlights the missing synapses in $p / r-1$ and $n r x-1$ mutants. Inset in (E) shows fainter proximal CLA-1 puncta in $n r x-1$ mutants. These were counted as synapses if their intensity was $>5 \%$ of the brightest CLA-1 peak. Scale bar $5 \mu \mathrm{m}$. (J, K) Quantifications of distance to first synapse (J) and synapse numbers $(\mathrm{K})$ in indicated genotypes. ${ }^{* *} p<0.001 ;{ }^{*} p<0.01 ;{ }^{*} p<0.05$ (Mann-Whitney $U$ test). (L) Domain structure of PLR-1 showing shy20 and hd129 allele. PA, protease-associated domain. TM, transmembrane domain. RING, RING finger. 
otherwise noted). RAB-3 vesicle clustering around active zones was normal in shy20 mutants (Figure S1), suggesting that shy 20 may be specifically related to Neurexin's function in defining synapse number and position rather than vesicle clustering. Using whole-genome sequencing and genomic rescue experiments, we mapped shy20 to the transmembrane E3 ubiquitin ligase PLR-1, homolog of mammalian RNF43 and ZNRF3 (Figure 1L). shy20 is a single point mutation that induces a premature stop codon (R188>Opal) before the transmembrane domain, suggesting that it is a strong loss-offunction allele. A second allele, plr-1(hd129) showed a similar phenotype to shy20 (not shown). Using rescue experiments, we determined that PLR-1 functions cell-autonomously in DA9 and that its function requires the RING domain, consistent with a role as a ubiquitin ligase (Figure $\mathbf{1} \mathbf{~ J , ~ K}$ ). Double mutants between $n r x-1$ and plr-1 showed a slight enhancement of the single mutant phenotypes, that was not statistically significant, suggesting that plr-1 and $n r x-1$ function in a common genetic pathway (Figure 11, J, K).

PLR-1 and its mammalian homologs specifically ubiquitinate Frizzled (Fz) receptors to downregulate Fz signaling (Hao et al., 2012; Koo et al., 2012; Moffat et al., 2014). In DA9, the Fz receptor LIN-17 has been shown to mediate anti-synaptogenic functions of its ligand LIN-44, but also to promote synapse-formation independently of LIN-44 (Klassen and Shen, 2007; Kurshan et al., 2018). We therefore speculated that the loss of synapses in plr-1 mutants was mediated by LIN-17. However, synapse loss in lin-17; plr-1 double mutants was similar to plr-1 mutants, indicating that LIN-17 does not function downstream of PLR-1 (Figure S2). Consistently, we did not observe changes in the subcellular distribution of LIN-17::YFP in p/r-1 mutants (Figure S2). These data are in agreement with a previous study in which LIN-17, unlike other Fz receptors, was not sensitive to PLR-1 overexpression (Moffat et al., 2014). We next examined a second Fz receptor, MIG-1, which does not have a known function in DA9. A MIG-1::GFP transgene expressed in DA9 was enriched in the dendrite but also localized to the axon. In the axon, MIG-1::GFP localized precisely to the region where synapses were lost in plr-1 mutants (Figure S3). This localization is consistent with the presence of source cells secreting Wnt/EGL20, MIG-1's ligand, in this region, and with the ability of Wnt/EGL-20 to control MIG-1 localization (Mizumoto and Shen, 2013; Pani and Goldstein, 2018). Importantly, mutations in mig-1 suppressed synapse loss in plr-1 mutants, strongly suggesting that PLR-1's function is to downregulate MIG-1 signaling to prevent synapse loss (Figure 1G, J, K). This suppression was reversed by expression of a MIG-1 transgene in DA9, indicating that MIG-1 signaling functions cell-autonomously (Figure $\mathbf{1} \mathbf{~ J , ~ K}$ ).

Since we found that $n r x-1$ and plr-1 function in a common pathway, we next examined $n r x-1$; mig-1 double mutants. In these animals, synapse loss was reduced compared to $n r x-1$ mutants, although only partially, despite the mig-1(e1787) allele being a putative null (Figure $\mathbf{1 H}, \mathbf{J}, \mathbf{K})$. These results indicate that MIG-1 signaling, regulated by PLR-1, acts either downstream or in parallel to Neurexin to antagonize synapse formation. Since NRX-1 also has MIG-1/Fz independent functions, such as RAB-3 vesicle clustering, it likely functions in parallel, rather than upstream, to MIG-1/Fz (Figure 2I).

\section{The atypical kinesin VAB-8/KIF26 is required for synapse formation downstream of NRX-1/Neurexin and MIG-1/Fz}

We next sought downstream targets of NRX-1 and MIG-1 that could mediate their effect on DA9 synapses. We focused on a second mutant, shy8, that displayed a similar phenotype to plr-1(shy20) in our microtubule minus-end screen (see below). Examination of GFP::CLA-1 and RFP::RAB-3 in shy 8 
mutants revealed loss of proximal synapses, but no vesicle clustering defects, similar to plr-1 mutants (Figure 2B, H, I, Figure S1). A postsynaptic marker, ACR-12::GFP, was also lost from the same region that showed presynapse loss, confirming the absence of proximal synapses in shy 8 mutants (not shown). shy8 mapped to vab-8, an atypical kinesin homologous to mammalian KIF26A and KIF26B. Kif26A functions in enteric and sensory neurons, where it regulates GDNF-Ret and FAK signaling, respectively. Kif26B functions in cell polarization and migration (Guillabert-Gourgues et al., 2016; Susman et al., 2017; Wang et al., 2018; Zhou et al., 2009) and mutations in human KIF26B are associated with progressive microcephaly and spinocerebellar ataxia (Nibbeling et al., 2017; Wojcik et al., 2018). In C. elegans, vab-8 is required for cell migration, axon extension of posteriorly growing neurons and GAP junction localization (Meng et al., 2016; Wightman et al., 1996; Wolf et al., 1998). DA9 grows anteriorly, and we did not detect DA9 axon growth or navigation phenotypes in vab-8 mutants.

The shy 8 mutation is a $153 \mathrm{bp}$ deletion and frameshift that leads to a premature STOP codon shortly after the motor domain (Figure 2F). A CRISPR-induced STOP codon in the same region (shy72) was indistinguishable from shy 8 and was used interchangeably. Comparison to previously isolated $v a b-8$ null alleles (ev411, gm84, e1017) or a trans-heterozygote with a genomic deletion encompassing the vab-8 region (shy8/arDf1) revealed similar phenotypic severity (not shown and Figure S4 for microtubule phenotypes) indicating that shy8 is a null allele. Expression of VAB-8 in DA9 rescued the Shy8 phenotype, revealing that it functions cell-autonomously to mediate synapse patterning (Figure 2G, H).

To gain further insight into how VAB-8 functions in DA9, we assayed the requirement for its predicted domains in rescue experiments. While full length VAB-8 robustly rescued synapse loss in $v a b-8$ mutants, constructs lacking the $\mathrm{C}$-terminal coiled-coils or the $\mathrm{N}$-terminal motor domain were inactive, consistent with a function as a motor (Figure 2G, H). Furthermore, mutating a conserved residue that is required for kinesin-1 motor domain binding to MTs (R278A) (Woehlke et al., 1997) was sufficient to render VAB-8 inactive (Figure 2G, H). These results indicate that microtubule-binding is required for VAB-8 function.

To test whether VAB-8/KIF26 functions downstream of Neurexin and Fz signaling, we conducted double mutant analysis. vab-8 (shy8) mutants did not enhance synapse loss of $n r x-1$ (wy778) and plr-1 (shy20) mutants, suggesting that the three proteins function in a common genetic pathway (Figure $\mathbf{2 C}$, G, H). Unlike the PIr-1 phenotype, which was suppressed by mutations in mig-1, mig-1; vab-8 double mutants retained the synapse-loss phenotype of vab-8 single mutants (Figure $\mathbf{2} \mathbf{G}, \mathbf{H}$ ). These observations are consistent with VAB-8 functioning downstream of MIG-1, and therefore also downstream of PLR-1. In agreement with this model, overexpression of VAB-8 in DA9 could ameliorate the phenotype of plr-1 mutants (Figure $\mathbf{2 G}, \mathbf{H}$ ). VAB-8 overexpression also suppressed synapse numbers and mispositioning defects in $n r x-1$ mutants (Figure 2D, E, G, H). These results indicate that VAB- 8 is required for patterning synaptic connectivity downstream of NRX-1 and MIG-1 (Figure 2I). A second Wnt receptor, LIN-17 also functions in DA9, where it plays both pro and anti-synaptogenic functions (Klassen and Shen, 2007; Kurshan et al., 2018). lin-17; vab-8 double mutants mostly resembled vab-8 mutants (Figure S2). Because LIN-17 has both pro and anti synaptogenic activities, we also tested double mutants between $v a b-8$ and the LIN-17 ligand, LIN-44, which only acts to antagonize synapse formation (Kurshan et al., 2018). vab-8 mutations suppressed the ectopic synapses that form in lin-44 mutants, further supporting the notion that VAB-8 is required for synapse formation in the DA9 axon (Figure S2). 
Figure 2
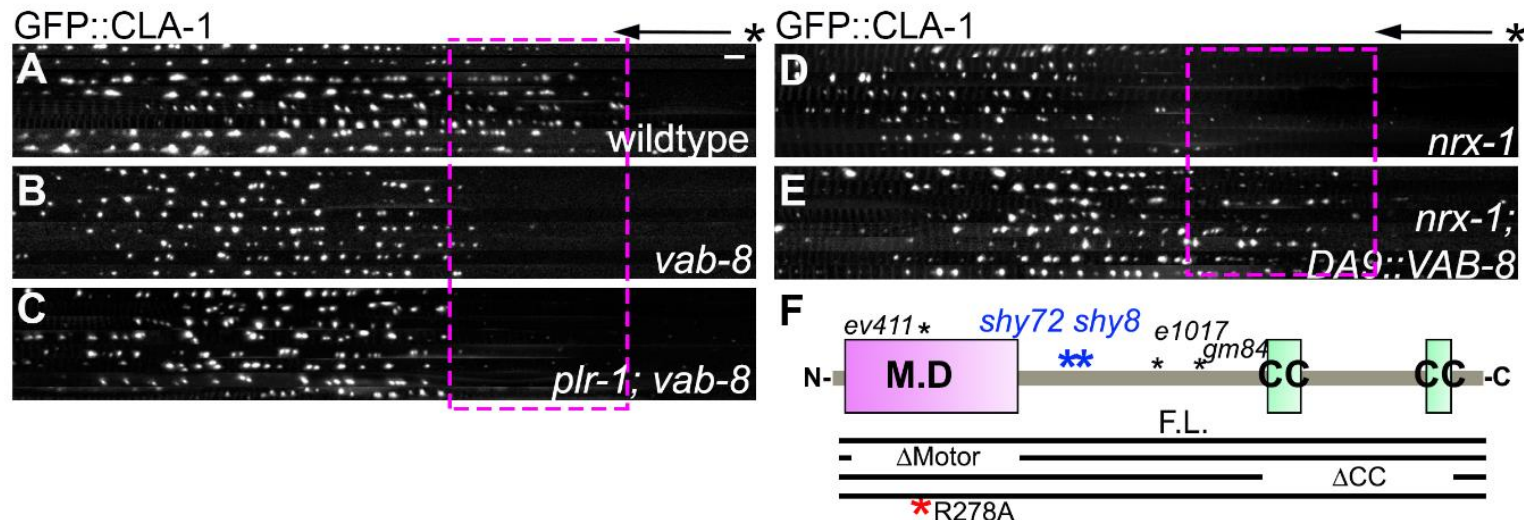

G

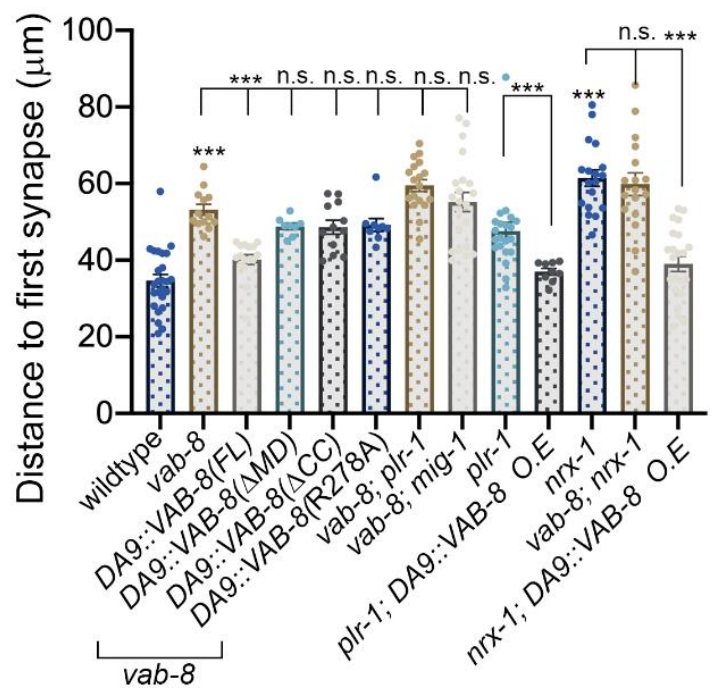

I

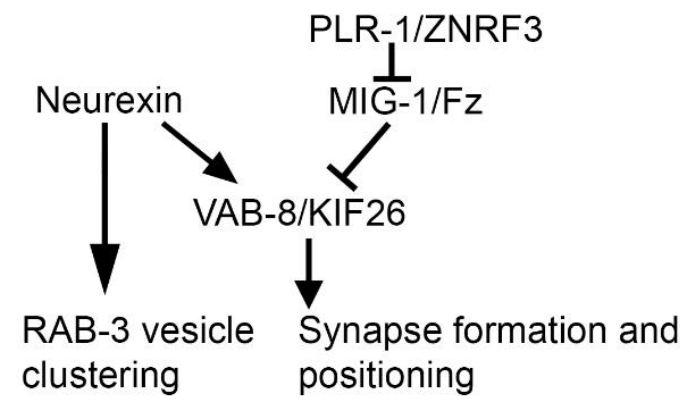

H

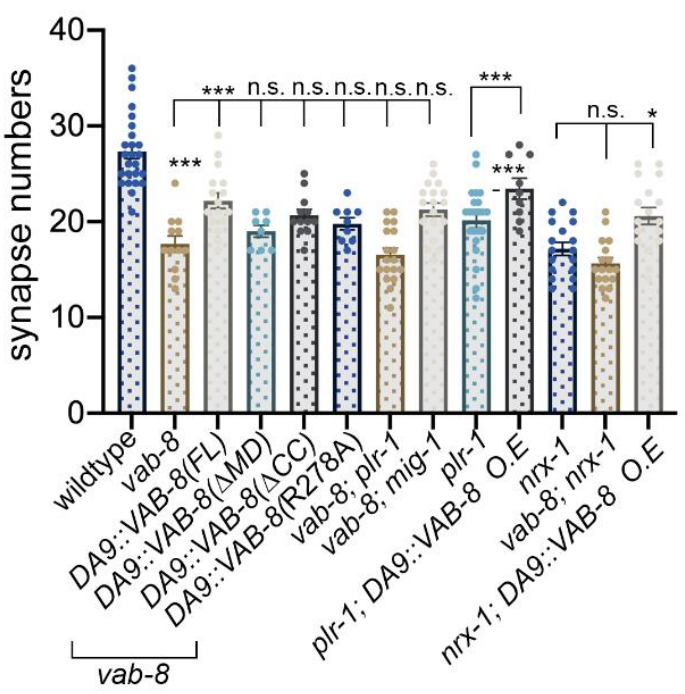

Figure 2: The atypical kinesin VAB-8/KIF26 control synapse formation downstream of NRX-1/Neurexin and MIG-1/Fz

(A-E) Alignments of 8 axons expressing GFP::CLA-1 from confocal images of the following genotypes: wildtype (A), vab-8 (B),

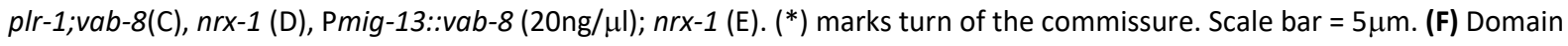
structure of $\mathrm{VAB}-8$ showing mutants alleles. Lower bars show rescue constructs. MD, motor domain. CC, coiled-coil domain. (G-H) Quantification of the distance from the turn of the commissure to the first synapse $(\mathrm{G})$ and synapse numbers $(\mathrm{H})$. ${ }^{* * *} p<0.001 ;{ }^{* *} p<0.01 ;{ }^{*} p<0.05$ (Mann-Whitney $U$ test). (I) Summary of genetic interactions among $n r x-1, v a b-8, p / r-1$ and mig-1. See text for details. 


\section{VAB-8 is a microtubule minus-end resident protein in neurons}

To gain insight into how VAB-8 controls synapses, we sought to investigate its subcellular localization. Endogenous VAB-8 tagged with 7XspGFP ${ }_{11}$ displayed discrete puncta when visualized with pan-neuronal spGFP $1-10$ (Figure S4). Although vab-8 mRNA is detected in DA9 at the L4 stage, its levels in this neuron were too low for visualizing the endogenous protein with a 7XspGFP 11 tag (Figure S4). A functional VAB-8::GFP transgene expressed in DA9 showed punctate localization throughout the axon (Figure $3 \mathrm{~A}$ ), consistent with the distribution we observed for endogenous VAB-8 in other neurons. We noticed that the frequency of VAB-8::GFP puncta is reminiscent of the frequency of microtubule minusends in DA9 (Yogev et al., 2016). Microtubules are polar structures, with a fast growing plus-end and a slow-growing minus-end. A large body of research identified many MT-plus-end proteins and their neuronal functions. Conversely, much less is known about regulation of MT minus ends, particularly in neurons (Akhmanova and Steinmetz, 2019; van de Willige et al., 2016). To test whether the VAB-8::GFP puncta we observe in the axon could correspond to MT-minus ends, we co-expressed VAB-8::GFP with RFP::PTRN-1/CAMSAP, which has been extensively validated as a minus-end marker (Feng et al., 2019; Goodwin and Vale, 2010; Jiang et al., 2014; Marcette et al., 2014; Meng et al., 2008; Wang et al., 2015). VAB-8::GFP puncta strongly colocalized with RFP::PTRN-1 puncta (Figure 3B), indicating that VAB-8 is minus-end resident protein.

We next asked whether VAB-8 directly binds to MT minus-ends or is recruited there. Interestingly, VAB-8 and KIF26 lack the residues required for ATP hydrolysis. KIF26 shows rigor-like binding to microtubules in co-sedimentation experiments, suggesting that it is likely immotile (Terabayashi et al., 2012; Wolf et al., 1998; Zhou et al., 2009). We used single-molecule TIRF microscopy to image VAB-8::mNeonGreen (mNG) on Taxol-stabilized microtubules in vitro and found that VAB8::mNG, was mostly immotile. We did not detect directional movement, but note that VAB-8::mNG can diffuse on the microtubule lattice (Figure 3D). VAB-8 was not enriched at the minus-end in vitro, suggesting that it is recruited to that location by other proteins in vivo. Consistent with a model in which VAB-8 diffuses on the microtubule to the minus-end, live imaging of VAB-8::GFP in DA9 did not reveal a motile vesicular component (Figure $\mathbf{3 C}$ ). To further test this model, we conducted FRAP experiments: VAB-8::GFP showed an immotile fraction of $\sim 73 \%$ and a recovery half-time of $\sim 13$ seconds (Figure $3 E$ ), consistent with a scenario of diffusion on the lattice and capture at the minus-end. We did not observe a significant difference between axon and dendrite, suggesting that the recruitment of VAB- 8 to minus ends is not locally regulated.

Finally, we examined whether known non-centrosomal microtubule minus-end proteins recruit VAB-8::GFP to minus-ends. VAB-8::GFP maintained its punctate pattern in ptrn-1 mutants, suggesting that $p t r n-1$ is not required for VAB-8::GFP localization to minus-ends (not shown). In the $C$. elegans germline and epidermis, a Ninein homolog, NOCA-1, localizes to minus-ends of non-centrosomal microtubules and functions redundantly with PTRN-1. A neuronal function for NOCA-1 has not been described (Wang et al., 2015). As noca-1 mutants are sterile, we examined homozygous progeny of heterozygous mothers, which likely contain maternally contributed noca-1. We observed a modest reduction in VAB-8::GFP puncta throughout the axon, with a stronger diffuse component. Interestingly, 
bioRxiv preprint doi: https://doi.org/10.1101/2021.03.22.436477; this version posted March 22, 2021. The copyright holder for this preprint (which was not certified by peer review) is the author/funder, who has granted bioRxiv a license to display the preprint in perpetuity. It is made available under aCC-BY-NC-ND 4.0 International license.

\section{Figure 3}
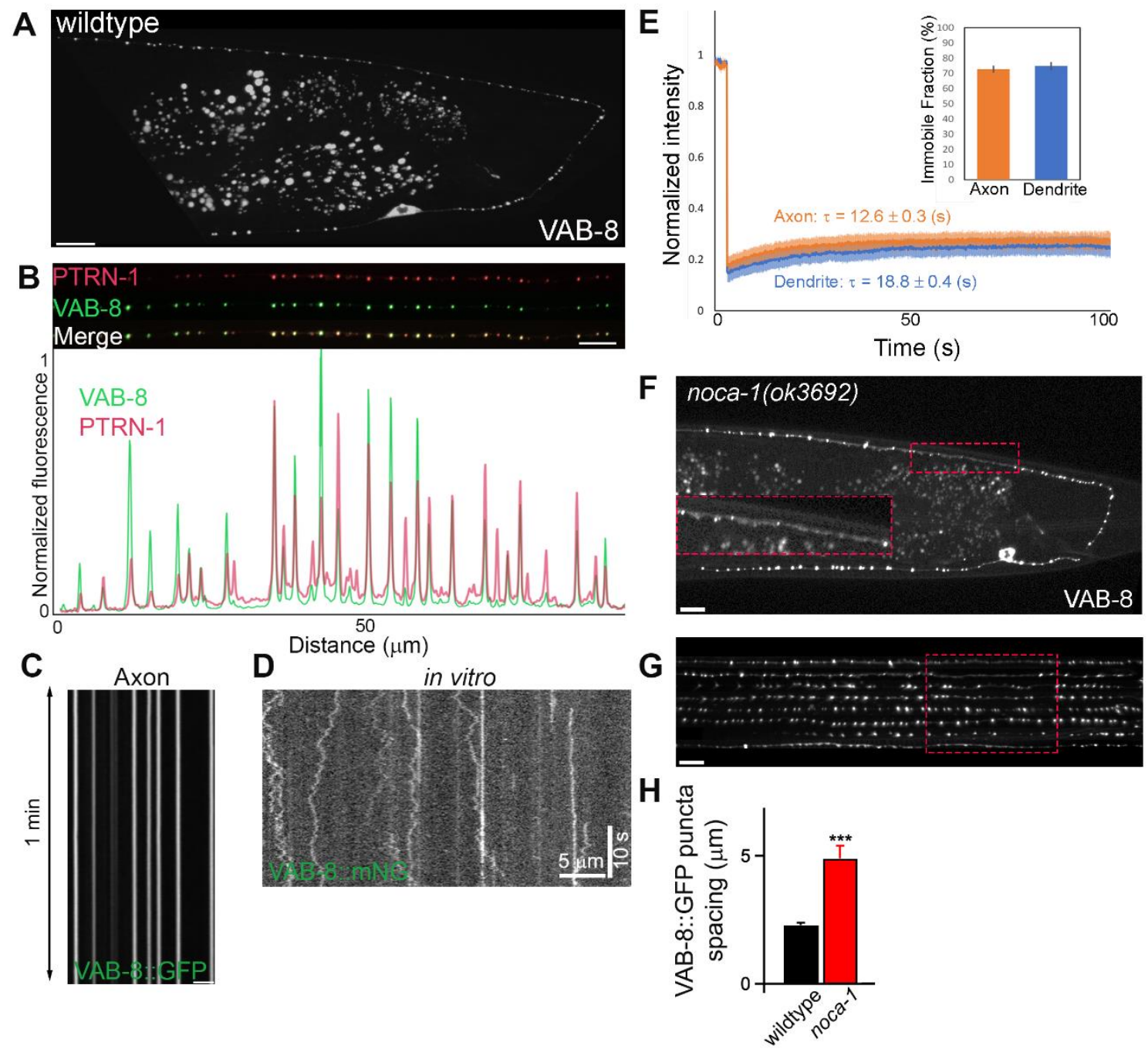

Figure 3: VAB-8 is a microtubule minus-end resident protein in neurons

(A) VAB-8::GFP in wildtype animals shows punctate staining in the axon and dendrite and a microtubule-like pattern in the cell body. Scale bars $=5 \mu \mathrm{m}$ in all panels. (B) VAB-8::GFP co-localizes with the microtubule minus-end marker RFP::PTRN-1/CAMSAP. Lower panel shows normalized fluorescence intensity plots. (C) Kymograph from a movie of VAB-8::GFP showing no discernible movement. (D) Kymograph from a TIRF movie showing VAB-8::mNG on microtubules in vitro. VAB-8 can diffuse on the lattice but does not show directional motility. (E) FRAP traces of VAB-8::GFP in the DA9 axon and dendrite reveal that most of the VAB-8 signal is immotile. $n=7$ per condition. (F) noca-1 is required for the localization of VAB-8. VAB-8::GFP puncta frequency is mildly reduced in noca-1(ok3692) mutants derived from heterozygous animals (noca-1 is required for viability in the embryo), with the strongest effect in proximal synapses. (G) Alignment of several axons from the same genotype as (F) shows that the region where VAB-8 loss occurs in noca-1 mutants is most consistently the proximal synapses. $(\mathrm{H})$ Quantification of the average spacing between VAB-8::GFP puncta in wildtype and noca-1 mutants. $n=20$ (wildtype) and 14 (noca-1). $* * * p<0.001$ (Mann-Whitnev $U$ test). 
the most prominent loss of VAB-8::GFP occurred at the proximal synaptic region, where $v a b-8$ and plr-1 mutants show loss of synapses (Figure 3F-H). An identical distribution of VAB-8::GFP was observed in ptrn-1; noca-1 double mutants (not shown). Taken together, these data indicate that $V A B-8$ is recruited to microtubule minus-ends throughout the neuron and is partially stabilized by NOCA-1/Ninein, particularly on the synaptic microtubules in the region controlled by Fz and Neurexin signaling.

\section{NRX-1/Neurexin and MIG-1/Fz control VAB-8/KIF26 accumulation on synaptic microtubule minus- ends}

Given that loss of NOCA-1 reduced VAB-8::GFP puncta most drastically in the synaptic domain, we next asked whether local signals in that area control VAB-8 abundance on minus-ends. plr-1 mutants, in which MIG-1/Fz signaling is increased, showed a drastic elimination of VAB-8 in the proximal synaptic domain (Figure 4B, G). Loss of VAB-8 in plr-1 mutants was more local and severe than in noca-1 mutants (compare Figure 4G with $\mathbf{3 H}$ ). Consistent with the synaptic phenotype, this effect could be significantly suppressed by mig-1 mutants (Figure 4C, G), suggesting that the excessive MIG-1/Fz signaling in p/r-1 mutants leads to local VAB-8 degradation. Although we cannot exclude that MIG/Fz interferes with VAB8 recruitment to minus-ends, we did not observe a significant increase in VAB-8::GFP between puncta. Proteasomal degradation of Kif26B has been previously observed during Kidney development and in migrating cells (Susman et al., 2017; Terabayashi et al., 2012). We next tested whether Neurexin plays a role in VAB-8 stability and found that in $n r x-1$ mutants, VAB- 8 was also strongly reduced in the synaptic region (Figure 4D, G). This reduction was mildly enhanced in plr-1; nrx-1 double mutants, but the enhancement was not statistically significant. The degradation of VAB-8::GFP in $n r x-1$ mutants was partially dependent on MIG-1, since VAB-8::GFP was restored in mig-1; $n r x-1$ mutants, although not completely (Figure 4F, G) (note that the mig-1(e1787) allele used is likely a null since it truncates MIG$1 / F z$ between its $3^{\text {rd }}$ and $4^{\text {th }}$ transmembrane domains). The effects of $p / r-1$ and $n r x-1$ on VAB- 8 stability are in agreement with the ability of overexpressed VAB- 8 to suppress the phenotypes of $n r x-1$ and $p / r-1$ mutants (Figure 2) and mirror the genetic interaction we observe between $n r x-1, p / r-1$ and mig-1 in synapse-patterning. Taken together, these results indicate that regulation of VAB-8 stability on synaptic microtubule minus-ends is an important mechanism for the control of synaptic connectivity by Neurexin and Fz signaling.

\section{VAB-8 is required for stabilizing minus-end proteins on synaptic microtubules}

We had originally identified VAB-8 and PLR-1 in a screen for mutants affecting the localization of the microtubule-minus-end marker PTRN-1::YFP in DA9. DA9 has a relatively low abundance of microtubules, similar to cerebellar parallel fibers (Harada et al., 1994), allowing to visualize individual minus-ends with PTRN-1::YFP (Yogev et al., 2016). In wildtype animals, axonal microtubule minus-ends appear with regular periodicity and accumulate an overall uniform level of PTRN-1::YFP (Figure 5A, D). In both $v a b-8$ and p/r-1 mutants, PTRN-1::YFP was reduced at the proximal synaptic area, leading to a local "gap" in the signal (Figure 5B-F). This effect was observed in several alleles of $v a b-8$ and plr-1, including nulls (Figure S5). Cell specific expression of VAB-8 and PLR-1 in DA9 rescued the phenotype, indicating that both proteins function cell-autonomously (Figure 5J). Interestingly, PTRN-1::YFP was not affected in nrx-1 mutants (not shown, see discussion). Together with VAB-8's localization, these results strongly suggest that VAB-8 functions at MT minus-ends. 


\section{Figure 4}
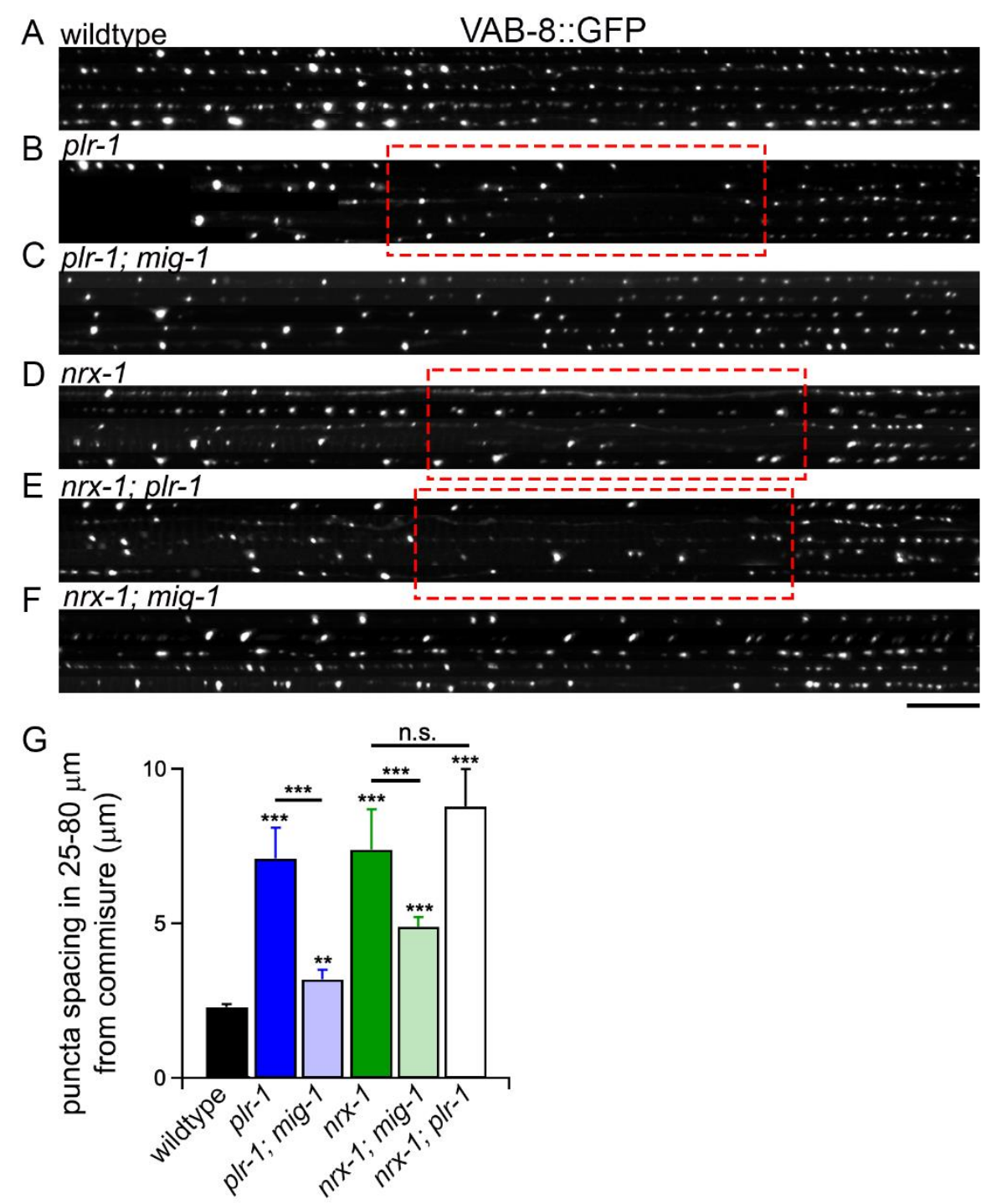

Figure 4: NRX-1/Neurexin and MIG-1/Fz control VAB-8 accumulation on synaptic microtubule minus-ends

(A-F). Confocal images of wildtype (A), plr-1(B), plr-1;mig-1(C), nrx-1(D), nrx-1;mig-1(E), nrx-1;mig-1(F) axons in DA9 expressing VAB$8:: G F P$. Five images from each genotype were straightened and aligned to the turn of the commissure $(*)$. Scale bar $=5 \mu \mathrm{m} .(\mathrm{G})$ Quantification of spacing between VAB-8::GFP puncta in the region between $25-80 \mu \mathrm{m}$ from the turn of the commissure. Note that similar to the synaptic phenotype, mutations in mig-1 partially suppress VAB- 8 degradation. $\mathrm{n}=10-20$ per genotype. ${ }^{* * *} p<0.001$; $* * p<0.01 ; * p<0.05$ (Mann-Whitney $U$ test). 
We next asked whether the reduction in PTRN-1::YFP indicates that no polymers start in the affected region, or alternatively reflects a local change in the abundance of minus-end associated proteins. We noted that MTs, labeled with a previously validated GFP::TBA-1/ $\alpha$-tubulin transgene (Yogev et al., 2016), were present in the region where PTRN-1 was reduced (Figure S6), suggesting a specific effect on minus-end proteins. To ask whether the dim PTRN-1 puncta in the affected region still localize to minus ends, we tested whether the averaged GFP::TBA-1/ $\alpha$-tubulin intensity around them rises to a peak, as would be expected if they localized to polymer ends. In wildtype animals, RFP::PTRN-1 puncta were associated with a rise in GFP::TBA-1 intensity, whereas locations midway between RFP::PTRN-1 puncta were not (Figure S7B). A similar behavior was observed in the affected region of both plr-1 and $v a b-8$ mutants (Figure S7C-F). These results indicate that VAB-8 is required for the normal accumulation of PTRN-1 on proximal synaptic microtubules, and not for the anchoring of MT minus-ends at this location.

In addition to PTRN-1, we also tested how loss of vab-8 or plr-1 affected NOCA-1, a Ninein homolog that localizes to acentrosomal microtubule minus-ends in the hypodermis and germline (Wang et al., 2015). Although PLR-1 is a transmembrane protein and is unlikely to directly affect microtubules, we analyzed plr-1 mutants because they allow to test the consequences of local loss of VAB- 8 from synaptic microtubules. NOCA-1d::GFP showed a punctate distribution similar to PTRN-1::YFP in wildtype animals (Figure 5G). In $v a b-8$ and plr-1 mutants, NOCA-1d::GFP was specifically reduced, but not completely eliminated, in the proximal synaptic domain, similar to the results obtained with PTRN-1::YFP (Figure 5G-I). Similarly to PTRN-1, NOCA-1 was not affected in $n r x-1$ mutants (not shown). Together, these results indicate that $v a b-8$ is locally required for the normal accumulation of other minus-end associated proteins on synaptic microtubules.

To determine the effect of VAB-8 loss on microtubule organization, we imaged animals harboring RFP::PTRN-1 and GFP::TBA-1/ $\alpha$-tubulin in wildtype and mutants. We first analyzed average microtubule length and numbers, using a method that we had previously developed and validated (Yogev et al., 2016, 2017). We observed little differences in the steady-state organization of microtubules between wildtype and $v a b-8$ or plr-1 mutants, further suggesting that VAB-8 does not regulate overall microtubule polymer abundance, length or distribution in neurons (Figure S7). To determine whether loss of vab-8 impinges on MT dynamics, we conducted live imaging on GFP::TBA$1 / \alpha$-tubulin. Visualizing tubulin rather than a plus-end binding protein enables us to measure both polymer growth but shrinkage (Figure 5K), which cannot be visualized by end-binding proteins. vab-8 and plr-1 mutants displayed a reduction in overall microtubule dynamicity, reflected by lower numbers of both growth and shrinkage events (Figure $\mathbf{5 L}$ ). This phenotype is reminiscent of the reduced microtubule dynamicity in ptrn-1 mutants (Richardson et al., 2014), in agreement with the local reduction of PTRN-1 in the affected region. We also observed changes in the velocity of growth and shrinkage, yet these effects were milder than the reduced dynamicity and were not consistent across plr-1 and vab-8 mutants (Figure $\mathbf{5 M}, \mathbf{N}$ ), suggesting that they do not play important roles in the effect of these mutations on synapses. These results identify VAB- 8 as a resident protein of microtubule minus ends, which is important to control the levels of other minus-end proteins and polymer dynamics of synaptic microtubules. 


\section{Figure 5}
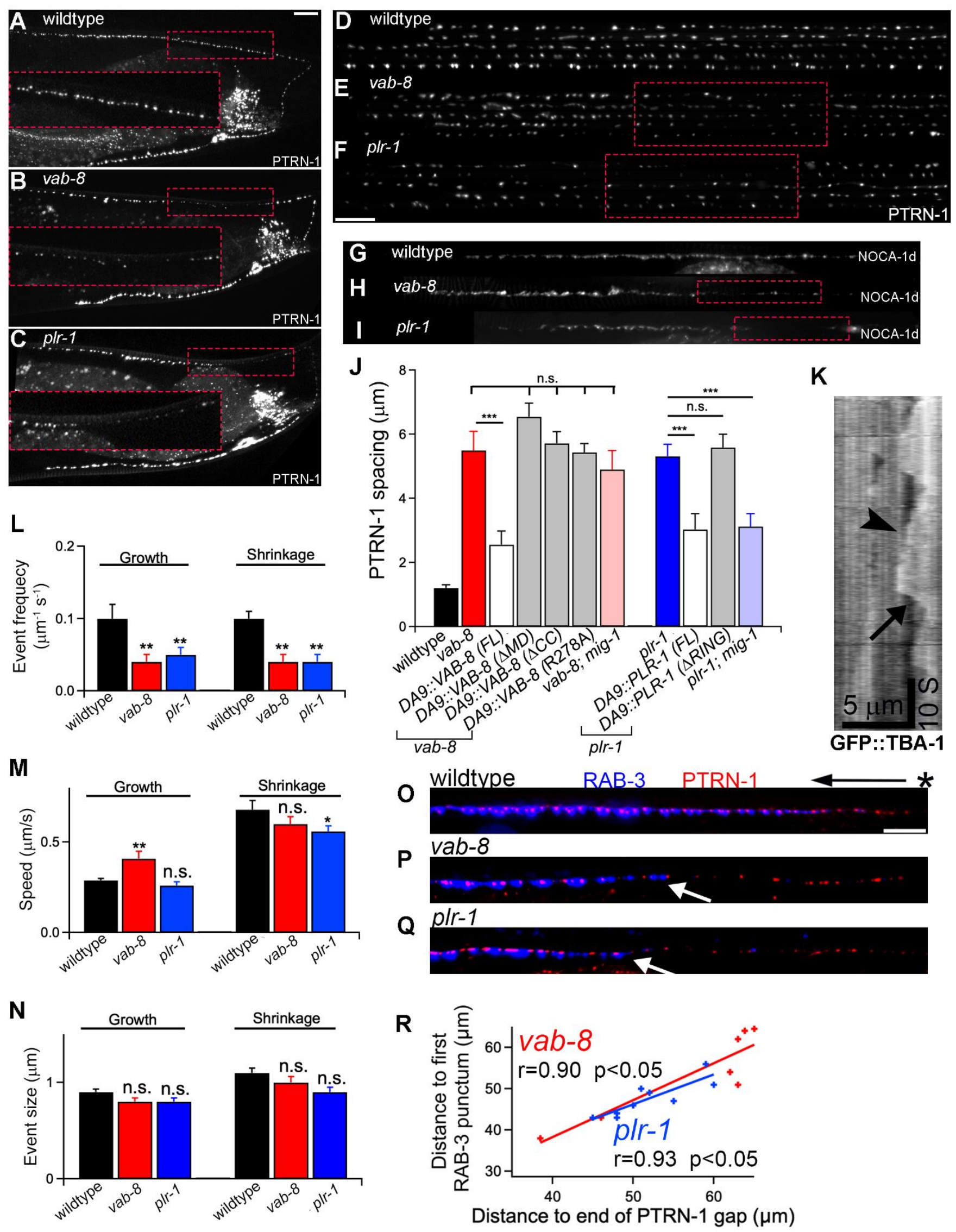
Figure 5. VAB-8 is required for stabilizing minus-end proteins on synaptic microtubules and cargo accumulates distally to the region affected in $v a b-8$ mutants

(A-C) Distribution of the microtubule minus-end marker PTRN-1::YFP in wildtype (A), vab-8 (B), and plr-1 mutants (C). Insets show the loss of PTRN-1 from the proximal synaptic region in the mutants. Scale bar $5 \mu \mathrm{m}$ in all panels. (D-F) Straightened axons from several animals per genotype highlight the stereotypic location where PTRN-1 is lost in the mutants. (G-I) A second microtubule minus-end associated protein, NOCA-1d, is specifically reduced in the proximal synaptic domain of $v a b-8$ (H) and plr-1 mutants (I) compared to wildtype (G). (J) Quantification of PTRN-1::YFP puncta spacing in the indicated genotypes. $\mathrm{N}=15-25$ per genotype. ${ }^{* *} p<0.001$ (Mann-Whitney $U$ test). (K-N) Microtubule dynamics in the DA9 axon were quantified on kymographs from movies of the microtubule marker GFP::TBA-1/ $\alpha$-tubulin (K). Arrowhead points to polymer growth and arrow points to shrinkage. Overall polymer dynamicity (number of growth and shrinkage events) is reduced in both vab-8 and plr-1 mutants (L). The speed of microtubule growth and shrinkage (M) in the mutants shows minor differences from wildtype whereas the size of these events $(\mathrm{N})$ is similar in all genotypes. $\mathrm{n}=20-30$ per genotype. ${ }^{* *} p<0.01$ (Mann-Whitney $U$ test). (O-Q) Co-expression of PTRN-1::YFP and and tdTomato::RAB-3 shows at least one microtubule minus-end in each synapse in wildtype $(\mathrm{O})$. In $v a b-8(\mathrm{P})$ and $p / r-1(\mathrm{Q})$ mutants, synaptic cargo accumulates distal to the region showing defects in PTRN-1. (R) Correlation between PTRN-1::YFP gaps and distance to first synapse/tdTomato::RAB-3 in vab8 (red) and $p / r-1$ (blue) mutants. $\mathrm{n}=12(v a b-8)$ and 20 (p/r-1). $r$ Pearson value and statistical significance (Student $t$ test) are showed.

\section{VAB-8 function at microtubule minus ends is related to its synaptic function}

To understand how VAB-8 function at microtubule minus ends might be related to its role in controlling synapse numbers and location, we first tested whether both functions require the same protein domains. Cell-specific rescue experiments showed that both VAB- 8 and PLR-1 act to maintain the normal distribution of PTRN-1 through the same domains that are required for synapse formation.

(Figure 5J). Furthermore, vab-8 and plr-1 mutants showed the same genetic interactions with each other and with mig-1 that were observed for the synaptic phenotype (Figure $5 \mathrm{~J}$ ). These results suggest that VAB-8 function at microtubule minus ends is related to its synaptic function.

One possibility is that loss of synapses is the primary defect, which then impairs the recruitment of PTRN-1 to minus-ends. To test this, we examined mutations in kinesin-3/KIF1A unc-104 (e1265), in which DA9 synapses are largely eliminated (Hall and Hedgecock, 1991; Ou et al., 2010). unc-104(e1265) mutants did not show a significant reduction in the recruitment of PTRN-1::YFP to discrete puncta, arguing that synapse loss is not the reason for the loss of PTRN-1 from minus-ends in vab-8 mutants (Figure S5). We next asked whether reduced PTRN-1 or reduced microtubule dynamicity could underlie the synaptic phenotype. However, DA9 synapses were largely unaffected in ptrn-1 mutants or in a $\beta$ tubulin/ tbb-2 (qt1) mutant that strongly suppresses microtubule dynamics in DA9 (Yogev et al., 2017) (Figure S5). We conclude that loss of synapses does not impair PTRN-1 recruitment and that reduced PTRN-1 and microtubule dynamicity do not cause the synaptic phenotype.

\section{VAB-8 promotes cargo pausing during axonal transport}

We next turned to the regulation of axonal transport as a possible link between VAB-8 function at microtubule minus-ends and synapses. Local regulation of cargo delivery from minus-ends into synapses is plausible because the frequency of minus-ends is higher than that of synapses, such that in wildtype DA9 every synapse is associated with at least one microtubule minus-end (Figure 50). We 
previously showed that during SVP transport, most kinesin-3/UNC-104 and dynein dependent runs terminate at microtubule ends (Yogev et al., 2016), raising the possibility that UNC-104 could be regulated at the plus-end and dynein at the minus-end. We distinguished three possible scenarios: first, If VAB-8 at minus-ends is indirectly affecting synapses, we would not expect the PTRN-1 gap to precisely correlate with the missing synapses in individual animals. Alternatively, if $\mathrm{VAB}-8$ functions to regulate cargo delivery during anterograde transport, synaptic material should accumulate proximal to the gap. Lastly, if VAB-8 mediates cargo delivery during retrograde transport, synapses should form distal to the gap. Co-labelling of RAB-3 and PTRN-1 showed a striking correlation between these markers in individual $v a b-8$ and $p / r-1$ animals (Figure 5P-R). Furthermore, synapses invariably formed distal to the gap, consistent with a failure in cargo delivery during retrograde transport (Figure 5P-R).

To test this model directly, we conducted live imaging of SVP transport. We photobleached the region affected by vab-8 mutants and imaged the SVP marker GFP::RAB-3 at $10 \mathrm{~Hz}$ (Figure 6A). We compared wildtype to $v a b-8$ and p/r-1 mutants, in which VAB-8 is specifically lost from synapses. Kymograph analysis of the movies showed minor changes in instantaneous velocity of cargo movement in plr-1 mutants, but not vab-8 mutants (Figure 6B). These results agree with the observation that VAB-8 is not detected as moving vesicular puncta and suggest that VAB-8 does not directly affect the ability of motors to transport cargo. We also did not detect significant changes to the overall ratio of anterograde to retrograde transport, suggesting that VAB-8 does not regulate overall cargo flux (Figure 6C).

We next examined cargo pausing during transport. Excessive pausing by kinesin-3/UNC-104 is associated with premature synapse formation in C. elegans, and pausing facilitates synaptic cargo retention in cultured hippocampal neurons (Guedes-Dias et al., 2018; Wu et al., 2013). In both plr-1 and vab-8 mutants, the number of pauses during transport was significantly decreased (Figure 6E). Furthermore, pauses were also shorter in either mutant compared to wildtype (Figure 6F). These results indicate that $\mathrm{VAB}-8$ functions to promote pausing during transport, potentially to facilitate cargo delivery. Consistent with VAB-8 localization to microtubule minus ends, pause frequency was mostly affected during dynein-driven retrograde transport (Figure 6G). In addition, we also observed that cargo run length was increased in both mutants (Figure 6D). Run-length increase is consistent with the reduction in pausing frequency and duration, which is expected to increase run length when pauses are shorter than our temporal resolution of $100 \mathrm{~ms}$. These results identify VAB-8 as a regulator of synaptic cargo transport, with its strongest effect being on pausing during retrograde transport.

\section{Synapse loss in vab-8 and $n r x-1$ mutants is due to excessive dynein-driven retrograde transport}

To test whether reduced pausing at microtubule ends is responsible for synaptic defects in vab-8 mutants, we asked whether compromising the processivity of axonal transport would rescue the synaptic phenotypes in vab-8 mutants. Although the specific localization of VAB- 8 to minus-ends and the more pronounced retrograde transport defects point to dynein as the relevant motor protein, our liveimaging data revealed reduced pause duration and increased run length also in the anterograde direction, probing us to also test the involvement of kinesin-3/UNC-104. To compromise transport in the anterograde direction we chose weak alleles of kinesin-3/UNC-104 and its activator ARL-8 (wy711 and tm2388, respectively) (Niwa et al., 2016; Wu et al., 2013) and to compromise retrograde transport we chose a weak allele of dynein heavy chain dhc-1 (js319) and its regulator NDEL1 nud-2 (ok949) (Koushika et al., 2004; Ou et al., 2010). We confirmed previous observations, that these single mutants do not 
Figure 6
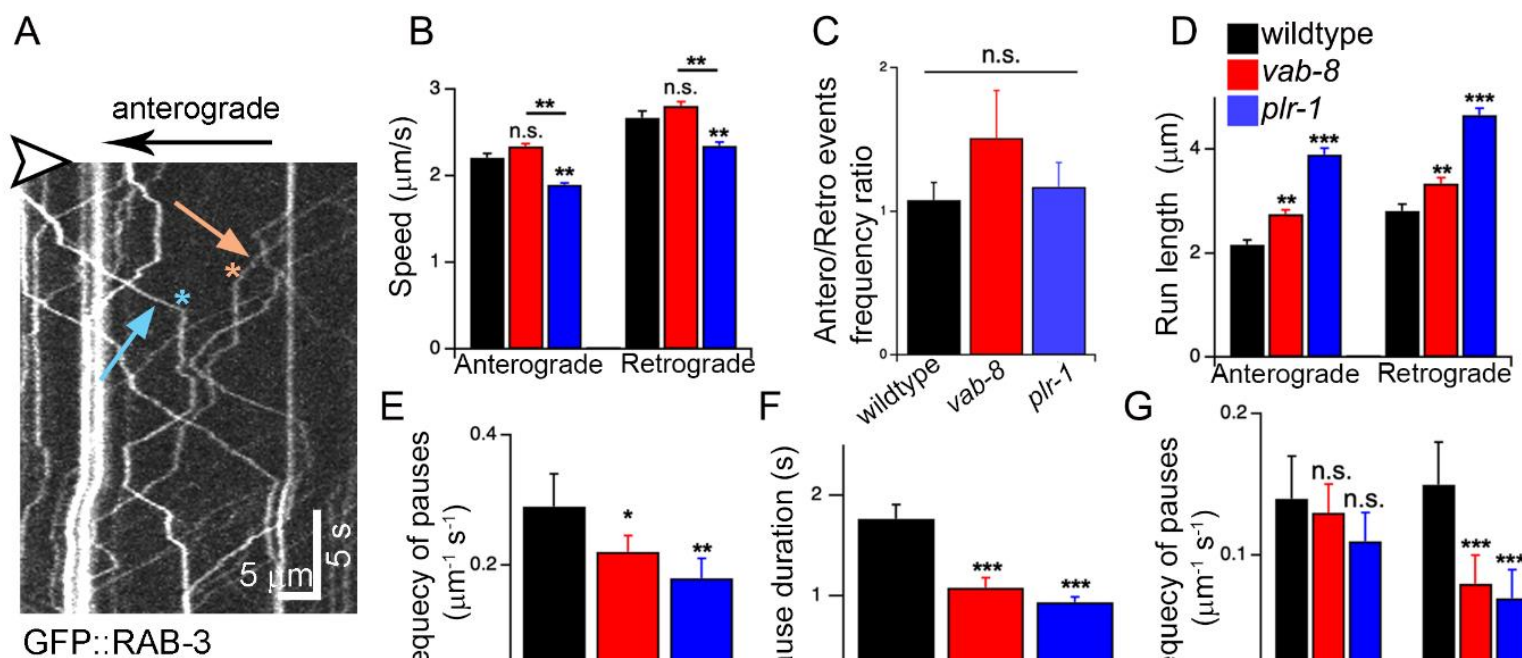

E
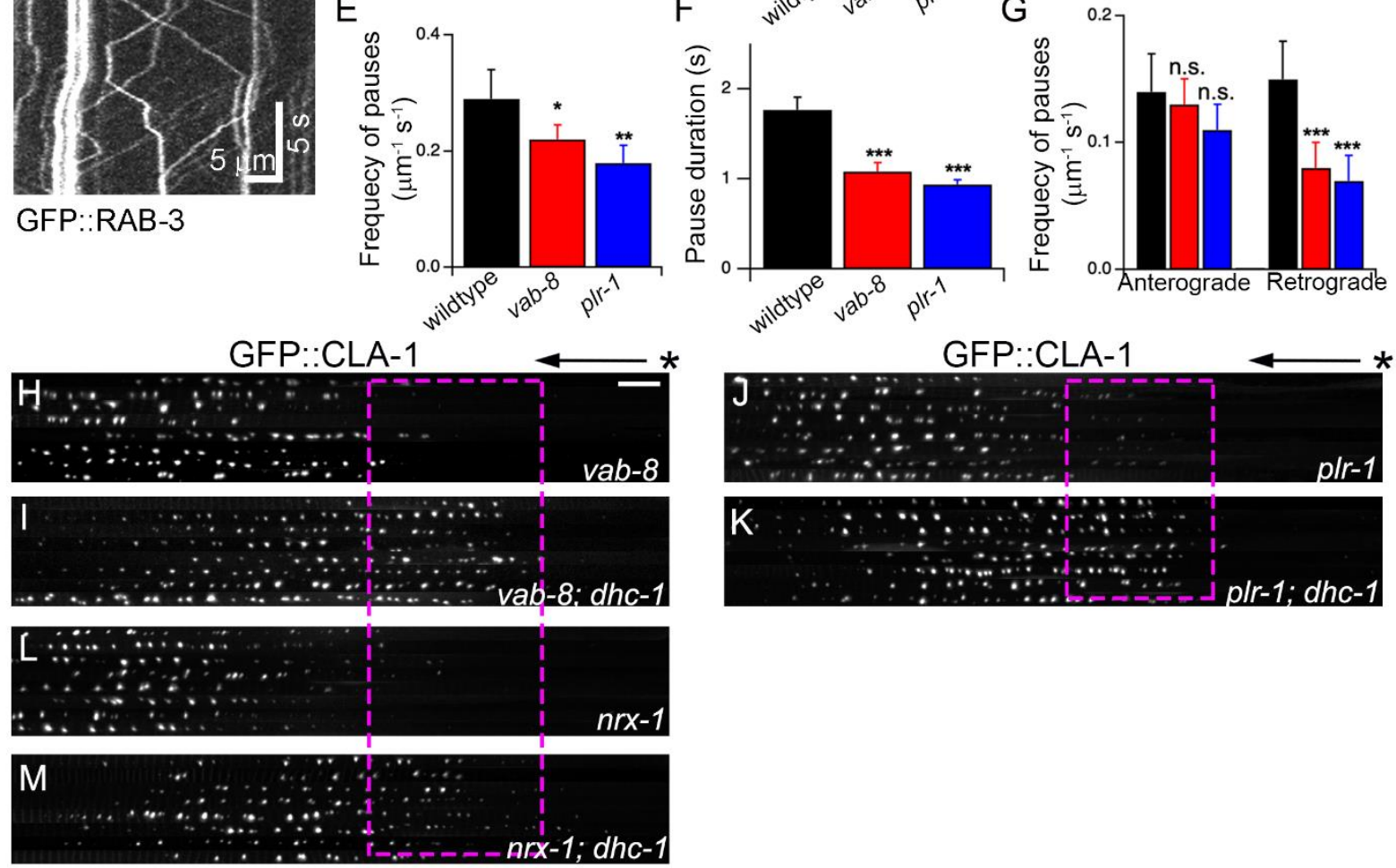

$\mathrm{N}$

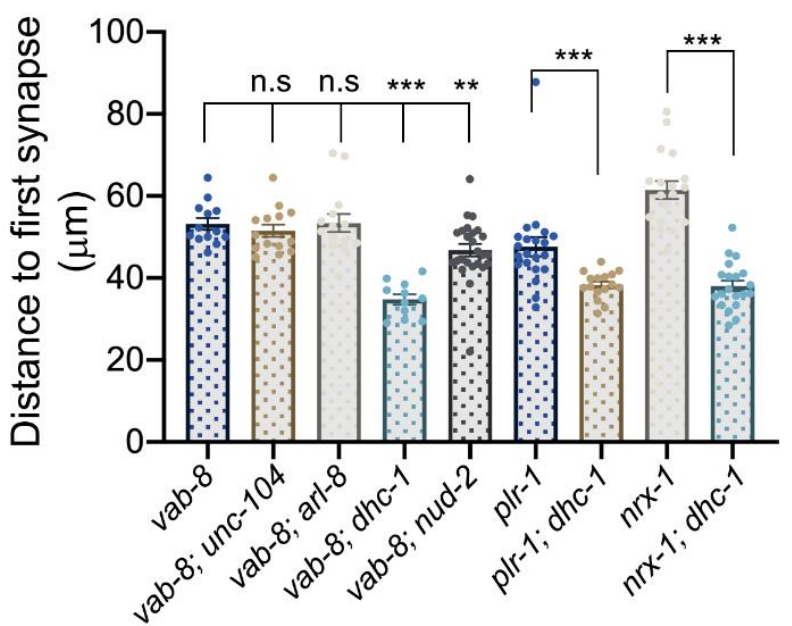


Figure 6: Synapse loss in vab-8 and $n r x-1$ mutants is due to excessive dynein-driven retrograde transport

(A) Example kymograph from a GFP::RAB-3 movie. Arrowhead indicates photobleaching, arrows and asterisks show runs terminating in pauses. (B-G) Quantification of SVP (GFP::RAB-3) transport in the DA9 axons from wildtype, vab-8 and plr-1 animals showing overall more processive transport in the mutants, particularly in the retrograde direction. Vesicle speed (B) or the ratio of anterograde to retrograde events (C) are similar in wildtype and mutants. Cargo run length (D) is increased in both mutants, whereas pause frequency is reduced (E) and pause duration increases (F). Pause frequency is specifically affected in the retrograde direction (G). $\mathrm{n}=20$-30 animals per genotype. ${ }^{* * *} p<0.001 ;{ }^{* *} p<0.01 ;{ }^{*} p<0.05$ (Mann-Whitney $U$ test). (H-M) Reducing dynein function rescues synaptic defects in $v a b-8, p / r-1$ and $n r x-1$ mutants. Images show 8 axons expressing GFP::CLA-1 aligned to the turn of the commissure. Genotypes: $n r x-1(\mathrm{H}), n r x-1 ; d h c-I(\mathrm{I}) ; v a b-8(\mathrm{~J})$, $v a b-8$; dhc-l (K), plr-1 (L), plr-1; dhc-I (M). Scale bar $5 \mu \mathrm{m}$. (N-0) Quantification of distance to first synapse/CLA-1 punctum $(\mathrm{N})$ and synapse numbers $(\mathrm{O})$ in the indicated genotypes. ${ }^{* * *} p<0.001{ }^{* *} p<0.01$; (Mann-Whitney $U$ test).

display overt synaptic defects, with the exception of $\operatorname{arl}-8$ (tm2388) which shows a mild posterior shift in synapse location (not shown) (Ou et al., 2010; Wu et al., 2013).

Double mutants between vab-8 and unc-104 (wy711) or arl-8 tm2388) displayed a similar phenotype to vab-8 single mutants (Figure $6 \mathbf{N}, \mathbf{0}$ ), suggesting that mis-regulated anterograde transport does not play causal roles in the steady-state $V a b-8$ phenotype. In striking contrast, reducing retrograde transport in vab-8 mutants, either with nud-2 (ok949) or more drastically with dhc-1 (js319), significantly rescued synapse numbers and positioning (Figure $6 \mathbf{H}, \mathbf{I}, \mathbf{N}, \mathbf{0}$ ). These results argue that VAB-8 regulates synapse formation and position by antagonizing dynein-driven transport.

Since we observed that VAB-8 functions downstream to PLR-1 and Neurexin, we extended the analysis to ask whether excessive retrograde transport also underlies synapse loss in these mutants. Previous live-imaging of cargo transport in $n r x-1$ mutants indeed revealed an increase in processivity that is highly reminiscent of our observations in vab-8 and plr-1 mutants (Kurshan et al., 2018). dhc1(js319) robustly suppressed the synaptic phenotypes of plr-1 (Figure 6J, K, N, 0). dhc-1(js319) also strongly suppressed the synapse-positioning defects of $n r x-1$ mutants and led to an increase in synapse numbers (Figure 6L-0). Taken together, our results identify the regulation of retrograde transport at microtubule minus-ends by VAB-8 as an important mechanism through which Neurexin and Frizzled signaling sculpt synaptic connectivity. 


\section{Discussion}

How the synaptogenic activity of cell-surface molecules promotes the local delivery of synaptic cargo undergoing long-range transport is poorly understood. Here we describe a role for the immotile kinesin VAB-8/KIF26 in linking Neurexin and Frizzled signaling to the delivery of synaptic cargo undergoing retrograde transport. We find that the levels of $\mathrm{VAB}-8$ at microtubule minus-ends are locally regulated by MIG-1/Fz and Neurexin signaling in a subset of presynaptic sites. In vab-8 mutants, or when VAB-8 levels are locally reduced in this region in plr-1 and $n r x-1$ mutants, those specific synapses are lost. Conversely, VAB-8 overexpression can ameliorate synapse-loss in p/r-1 and $n r x-1$ mutants, suggesting that it is an important mediator of PLR-1's and Neurexin's presynaptic function. In vab-8 mutants, $n r x-1$ mutants (Kurshan et al., 2018) and plr-1 mutants, retrograde axonal transport becomes overly processive. Importantly, synapse-loss in these mutants can be rescued by compromising retrograde transport. Together, these results strongly support a model in which the pro- and antisynaptogenic activities of Neurexin and Fz converge on VAB-8/KIF26 to regulate the delivery of synaptic cargo undergoing retrograde transport from microtubule minus-ends. This model is further supported by the localization of both paused dynein (Yogev et al., 2016) and VAB-8/KIF26 to microtubule minusends, and by the specific formation of synapses distally to the affected region in vab-8 mutants.

\section{Synaptogenic activity promotes the local delivery of cargo undergoing transport}

Synapse formation entails the recruitment of actin, scaffolding and active zone molecules, and vesicle precursors (Rizalar et al., 2021; Südhof, 2018). Although binding partners for Neurexin's intracellular domain have been identified, how Neurexin functionally promotes presynapse formation is not yet understood. Neurexin's intracellular domain binds the scaffolding proteins CASK and MINT, and Neurexin clustering can promote synaptic vesicle recruitment (Biederer and Südhof, 2000; Biederer and Sudhof, 2001; Dean et al., 2003; Hata et al., 1996; Mukherjee et al., 2008). In Drosophila, Neurexin binds the active zone molecule SYD-1 and the actin binding protein Spinophilin (Muhammad et al., 2015; Owald et al., 2012). Our results identify the regulation of cargo delivery during axonal transport as an important facet of Neurexin's synaptogenic activity: synapse loss in $n r x-1$ mutants is rescued by VAB-8 overexpression or by reducing retrograde transport. Wnt/Fz signaling is also well known to regulate synaptogenesis, although unlike Neurexin, it often plays inhibitory roles (He Chun-Wei et al.). Synaptic Fz signaling in Drosophila is important for proper cytoskeletal organization, but exactly how those relate to transport is unclear (Lüchtenborg et al., 2014; Miech et al., 2008; Sugie et al., 2015). Our data show that degradation of VAB-8/KIF26 is a mechanism for antagonizing synapse formation by MIG-1/Fz and reveal that VAB-8 is required for ectopic synapse formation in lin-44/Wnt mutants. However, they do not address how Fz/LIN-17 positively regulates synapse-formation. Importantly, linking VAB-8 to synaptic cargo delivery from microtubule ends provides a plausible mechanism for how the control of cytoskeletal proteins by Wnt/Fz signaling regulates synaptogenesis.

\section{Transport pausing at microtubule minus-ends facilitates cargo capture by synapses}

Most synaptic proteins are synthesized in the cell body and rely on long-range microtubuledependent transport to reach synapses (Ahmari et al., 2000; Kreutzberg, 1969). Local cargo delivery, the last step in transport prior to retention/capture at a synaptic site, is still poorly understood. Live imaging of DCVs revealed that their delivery occurs during both anterograde and retrograde transport and is promoted by synaptic activity (Bharat et al., 2017; Cavolo et al., 2016; Wong et al., 2012). However, how 
delivery is regulated by synaptogenic signals is still elusive. We found that VAB-8/KIF26 is locally controlled by Neurexin and Frizzled signaling to promote cargo delivery to a specific subset of synapses. Furthermore, in the absence of $v a b-8$ these synapses are missing, highlighting the importance of local regulation of cargo delivery as a mechanism to control synapse formation. Interestingly, distal synaptic boutons in DA9 were not affected by vab-8, nrx-1 or plr-1 mutations, raising the possibility that delivery during anterograde transport plays a more prominent role in those distal synapses.

DCV capture/retention is promoted by interactions of kinesin-3/KIF1A with the scaffolding molecules liprin- $\alpha$ and TANC2 at dendritic spines, and DCV dissociation from kinesin-3 is enhanced by JNK-dependent phosphorylation of Synaptotagmin-4 at presynaptic sites (Bharat et al., 2017; Stucchi et al., 2018). Because cargo needs to bypass many en passant synapses to ensure uniform distribution among all sites, these - and potentially other - synaptic capture mechanisms operate at a low probability ( $1 \%$ for SVPs in cultured hippocampal neurons (Guedes-Dias et al., 2018)). Hence, pausing during transport is likely important to allow synaptic capture mechanisms to engage with motor-cargo complexes.

Kinesin-3 and dynein pause on microtubule plus and minus-ends, respectively (Cai et al., 2009; Soundararajan and Bullock, 2014; Tan et al., 2018; Yogev et al., 2016). kinesin-3 shows lower affinity for the GTP cap, and therefore an association between dynamic plus-ends and synapses was proposed to promote cargo delivery by kinesin-3 (Guedes-Dias et al., 2018). This suggests that the increased anterograde processivity of SVPs that we observe in the absence of VAB-8 may stem from the reduced plus-end dynamics in the mutants. Alternatively, it could be a by-product of losing synaptic capture mechanisms, as proposed in the case of $n r x-1$ mutants (Kurshan et al., 2018). Importantly, suppression of Vab-8 phenotypes by $d h c-1$ and nud-2 but not unc-104 and arl-8 allows us to distinguish which dynamic transport parameters are causal for the steady-state synaptic phenotype. The conclusion that overly processive retrograde transport drives synapse loss is also supported by the association between minus ends and synapses and the accumulation of cargo distally, rather than proximally, to the affected region in the mutants. We propose that reduced pausing, which is mostly observed in the retrograde direction, is the most relevant parameter because of the inefficiency of synaptic capture mechanisms, which may be unable to engage with moving cargo.

\section{VAB-8/KIF26 function at microtubule minus ends}

How does VAB-8 regulate retrograde transport and the accumulation of minus-end proteins? Our TIRF imaging suggests that VAB-8 does not directly identify the structure of the minus-end but is recruited to these locations, at least partially by NOCA-1. Since in vab-8 mutants the reduction in PTRN-1 and NOCA-1 - both of which directly bind minus ends (Goodwin and Vale, 2010; Meng et al., 2008; Wang et al., 2015) - is restricted to the synaptic region and is not accompanied by a redistribution of these proteins to other parts of the neuron, we favor a model where synaptic VAB-8 protects other minus-end proteins from degradation. Consistently, proteasomal degradation controls Kif26b levels during kidney development and in cultured mesenchymal cells exposed to Wnt 5A-Ror signaling (Susman et al., 2017; Terabayashi et al., 2012). Interestingly, in the absence of VAB-8 and MIG-1, degradation of PTRN-1 was still observed at proximal synapses, although it was reduced. Furthermore, while VAB- 8 was strongly reduced in $n r x-1$ mutants, PTRN-1 and NOCA-1 were not. Together, these observations suggest that an additional signal regulates the composition of minus-end protein complexes in the synaptic region, possibly downstream of Neurexin. This scenario is also consistent with 
the observation that mig-1 and $d h c-1$ mutants, as well as VAB-8 over-expression, rescue $n r x-1$ mutations to a lesser extent than that observed for plr-1. Precisely how VAB-8 promotes pauses of dynein remains to be elucidated. Kif26a was proposed to anchor and regulate kinase pathways on the microtubule lattice (Wang et al., 2018; Zhou et al., 2009), raising the possibility that VAB-8 at minus ends may localize a kinase that could phosphorylate dynein or its cargo. A potential candidate would be JNK, which is required for DCV capture and SVP clustering (Bharat et al., 2017; Wu et al., 2013). It would be interesting to test whether VAB-8 could facilitate interactions between JNK and dynein at synapses. 


\section{Methods}

\section{Strains and maintenance}

All C. elegans strains were cultured on nematode growth medium plates seeded with $E$. coli OP50 as previously described (Brenner, 1974). Animals were grown at $20^{\circ} \mathrm{C}$ prior to experiments/injections and at room temperature afterward. The N2 Bristol strain was as wildtype for outcrosses. C. elegans strains used are listed in S1 Table.

\section{Genetic screen}

We performed a visual forward F2 genetic screen of 2,000 haploid genomes. Ethyl methanesulfonate (EMS) was used to induced random germline mutations in animals carrying the wyls802 [Pitr-1::PTRN-1::YFP] marker. Mutagenized worms were scored for changes in YFP::PTRN-1a in DA9. Homozygous mutants were rescued and outcrossed 5 times with N2 males prior to phenotypic analysis and whole-genome sequencing. Genomic DNA was purified with Phenol/ $\mathrm{CHCl}_{3}$ (InvitrogenThermo Fisher) and precipitated with ethanol/ $\mathrm{NaCH}_{3} \mathrm{COO}$ (Sigma-Aldrich). DNA was resuspended and solubilized in DNAse-free water (Sigma-Aldrich) and sequenced on Illumina Hi-Seq machines at the Yale Center for Genome Analysis. Sequencing data was analyzed by using tools on Galaxy Project. Briefly, reads were aligned against $C$. elegans N2 genome as a reference. Variants were called and filtered to isolate strain-specific single-nucleotide and indel mutations. The causality of mutations in $v a b-8$ and $p / r$ 1 for the phenotypes was confirmed by rescue experiments, testing additional alleles and generation of additional mutants using CRISPR.

\section{Cloning and constructs}

All plasmids were generated by Gibson Assembly and confirmed by Sanger sequencing. cDNA was prepared from C.elegans lysates according to standard procedures. Sequences and plasmids are freely available upon request.

\section{CRISPR/Cas9 genome editing}

We used CRISPR to generate shy 72 and shy 73 mutations in vab-8 near the shy 8 mutation in order to confirm the causality of mutations in that region to the $V a b-8$ phenotype and in order to produce double mutants $v a b-8, n r x-1$ since the two genes are too close to each other for generating recombinants. We followed the protocol of (Dokshin et al., 2018). Cas9 protein was purchased from IDT. sgRNA was synthesized using EnGen kit from NEB using the following primer:

ttctaatacgactcactatagTCAATCCCTCCCATGCTCCGgttttagagctaga. The following single strand repair template was used to induce STOP mutations:

AACACACAACTGCCACGACGGTTGTATTCATTCAATCCCTCCCATGCTATAAGGATCCCTAATTAACCGTAGACACA CGCCATTCCTCTCTGCATCACTGAAACTGTACGACGA. Sanger sequencing was used to confirm the repair.

\section{Fluorescence microscopy}

Animals were synchronized as L4 larvae and grown for an additional 20-24 hrs at 20 degrees to reach adulthood. We immobilized worms on agar pads with 5-10 mM Levamisole in M9 buffer for standard fluorescence imaging. Images were acquired through an HC PL APO 63x/1.40NA OIL CS2 objective or a PL APO 40x/1.30NA CS2 objective built on a Laser Safe DMi8 inverted microscope (Leica) equipped with a VT-iSIM system (BioVision). Images were captured with an ORCA-Flash4.0 camera 
(Hamamatsu) and controlled by MetaMorph Advanced Confocal Acquisition Software Package. For any given set of data, the same settings (laser power, exposure time, and gain) were used.

For time-lapse imaging, animals were incubated in levamisole $(0.5 \mathrm{mM})$ in $\mathrm{M} 9$ at room temperature for 5 min prior to mounting on 10\% agarose slide in M9 without paralytics, ensuring a low concentration of levamisole that was determined not to produce artifacts. Movies were collected on an Olympus BX61 microscope equipped with a Hamamatsu ORCA-Flash4.0 LT camera at 3 or $10 \mathrm{~Hz}$ (microtubules and synaptic vesicles respectively) for $3 \mathrm{~min}$. Image acquisition was monitored by Volocity 2.0 (Quorum technologies). For any given set of data, the same settings (laser power, exposure time, and gain) were used. FRAP of GFP::RAB-3 and VAB-8::GFP was achieved by a 50 milliseconds pulse of 488-laser with a Perkin-Elmer Photokinesis unit.

\section{Fluorescence quantification}

Raw data were exported for analysis on ImageJ or MATLAB. Kymographs were generated using the ImageJ KymoResliceWide plugin following correction for animal movement using the StackReg plugin. Traces on kymographs were analyzed manually and data was exported to Excel and Igor Pro 8 for further analysis. To quantify the number of synapses, a line-scanning method along the axon was used to obtain the GFP::CLA-1 fluorescent profile. We used the same criterion as (Kurshan et al., 2018) to count a peak in the signal as a synapse ( $>5 \%$ of the maximum) for consistency with previous literature. Note that this criterion usually leads to the scoring of the fainter CLA-1 puncta in $n r x-1$ mutants (highlighted Figure 1) as synapses. VAB-8::GFP axonal localization was resolved by drawing a region of interest (ROI) around the axon. Based on the intensity histogram, a default threshold was applied to the ROI to create a binary mask. The mask was firstly treated with the built-in Watershed segmentation algorithm to better define nearby puncta. Lastly, the number of puncta and their intensity were assessed by using the Analyze Particle function provided in ImageJ. PTRN-1 puncta spacing was quantified by measuring the distance between neighboring YFP::PTRN-1a puncta in the axonal region of interest. A custom MATLAB code (available upon request) was used to analyze the distribution of GFP::TBA-1 around RFP::PTRN-1 peaks. Briefly, the code determines the location of PTRN-1 peaks and averages GFP::TBA-1 in a 15-pixel region around each peak. As a control, the midway point between two peaks is used. To average the signal across multiple animals, the "on peak" traces were normalized by "off peak traces" in order to prevent variability in marker expression to impact the analysis. Axonal microtubule organization and minus-end analysis was done using a previously described and validated MATLAB code (Yogev et al., 2016), which is available upon request.

\section{Single-molecule motility assays}

To generate pMT-VAB-8-mNeonGreen plasmid, VAB-8 was amplified by PCR from Pmig13::vab-8 and subcloned into pMT-mNeonGreen vector.

Drosophila S2 cells were cultured in Schneider's Drosophila medium (Gibco) supplemented with $10 \%$ (vol/vol) FBS (HyClone) at $26^{\circ} \mathrm{C}$. Plasmid for expression of VAB-8-mNeonGreen in the pMT vector was transfected into $\mathrm{S} 2$ cells using Cellfecinll (Invitrogen) according to manufacturer's instructions. Expression of VAB8-mNeonGreen was induced by adding $1 \mathrm{mM} \mathrm{CuSO}_{4}$ to the medium after $4-5 \mathrm{~h}$ transfection. To prepare cell lysate for single molecule assays, $\mathrm{S} 2$ cells were harvested after $48 \mathrm{~h}$ posttransfection. The cells were centrifuged at low-speed at $4^{\circ} \mathrm{C}$. The cell pellet was washed with PBS buffer and resuspended in ice-cold lysis buffer $(25 \mathrm{mM}$ HEPES/KOH, $115 \mathrm{mM}$ potassium acetate, $5 \mathrm{mM}$ sodium 
acetate, $5 \mathrm{mM} \mathrm{MgCl} 2,0.5 \mathrm{mM}$ EGTA, and 1\% Triton X-100, pH 7.4) freshly supplemented with $1 \mathrm{mM}$ ATP, $1 \mathrm{mM}$ PMSF, and protease inhibitors (Sigma-Aldrich). After the cell lysate was clarified by centrifugation at full-speed at $4^{\circ} \mathrm{C}$, aliquots of the supernatant were snap frozen in liquid nitrogen and stored at $-80^{\circ} \mathrm{C}$ until further use.

HiLyte647-labeled microtubules were polymerized from purified tubulin including $10 \%$ Hily647labeled tubulin (Cytoskeleton) in BRB80 buffer ( $80 \mathrm{mM}$ Pipes/KOH pH 6.8, $1 \mathrm{mM} \mathrm{MgCl}$, and $1 \mathrm{mM} \mathrm{EGTA}$ ) supplemented with $1 \mathrm{mM} \mathrm{GTP}$ and $2.5 \mathrm{mM} \mathrm{MgCl}_{2}$ at $37^{\circ} \mathrm{C}$ for $30 \mathrm{~min} .20 \mu \mathrm{M}$ taxol in prewarmed BRB80 buffer was added and incubated at $37^{\circ} \mathrm{C}$ for additional $30 \mathrm{~min}$ to stabilize microtubules. Microtubules were stored in the dark at room temperature for further use. A flow cell ( $\sim 10 \mu$ l volume) was assembled by attaching a clean \#1.5 coverslip (Fisher Scientific) to a glass slide (Fisher Scientific) with two strips of double-sided tape. Polymerized microtubules were diluted in BRB80 buffer supplemented with $10 \mu \mathrm{M}$ taxol and then were infused into flow cells and incubated for $5 \mathrm{~min}$ at room temperature for nonspecific adsorption to the coverslips. Subsequently, blocking buffer $[1 \mathrm{mg} / \mathrm{ml}$ casein and $10 \mu \mathrm{M}$ taxol in P12 buffer] was infused and incubated for $5 \mathrm{~min}$. Finally, kinesin motors (0.5-2 $\mu$ l cell lysate) in the motility mixture [ $2 \mathrm{mM}$ ATP, $3 \mathrm{mg} / \mathrm{ml}$ casein, $10 \mu \mathrm{M}$ taxol, and oxygen scavenging in P12 buffer] was added to the flow cells. The flow-cell was sealed with molten paraffin wax.

Images for single molecule assay were acquired by TIRF microscopy using an inverted microscope Ti-E/B (Nikon) equipped with the perfect focus system (Nikon), a 100× 1.49 NA oil immersion TIRF objective (Nikon), three 20-mW diode lasers (488 nm, $561 \mathrm{~nm}$, and $640 \mathrm{~nm}$ ) and an electron-multiplying charge-coupled device detector (iXon X3DU897; Andor Technology). Image acquisition was controlled using Nikon Elements software and all assay were performed at room temperature. Images were acquired continuously at $200 \mathrm{~ms}$ per frame for $40 \mathrm{~s}$. Maximum-intensity projections were generated and the kymographs were produced by drawing along tracks of motors (width= 3 pixels) using ImageJ.

\section{Statistical analysis}

Statistical analysis was performed on Igor Pro 8, Prism 9 (GraphStats), and SPSS (IBM). Data were considered significant at $p \leq 0.050$ and are plotted as the means with SEM. Each data set was compared using a Mann-Whitney $U$ test unless otherwise specified. A Student $t$ test was used to compare data groups when both passed the normality test (Kolmogorov-Smirnov).

\section{Strain list}

\begin{tabular}{|c|l|}
\hline $\begin{array}{c}\text { Strain } \\
\text { name }\end{array}$ & \multicolumn{1}{c|}{ Genotype } \\
\hline TV18675 & wyls685 [Pmig-13::cla-1::gfp, Pmig-13::rab-3::tdTomato, Podr-1::gfp] \\
\hline MTS473 & plr-1(shy20); wyls685 [Pmig-13::cla-1::gfp, Pmig-13::rab-3::tdTomato, Podr-1::gfp] \\
\hline MTS942 & $\begin{array}{l}\text { plr-1(shy20); wyls685 [Pmig-13::cla-1:::gfp, Pmig-13::rab-3::tdTomato, Podr-1::gfp]; } \\
\text { shyEx241 [Pmig-13::plr-1::scarlet, Punc-122:::rf] }\end{array}$ \\
\hline MTS900 & $\begin{array}{l}\text { plr-1(shy20); wyls685 [Pmig-13::cla-1::gfp, Pmig-13::rab-3::tdTomato, Podr-1::gfp]; } \\
\text { shyEx234 [Pmig-13::plr-1(A317-358::scarlet), Punc-122::rfp] }\end{array}$ \\
\hline MTS962 & nrx-1(wy778), wyls685 [Pmig-13::cla-1::gfp, Pmig-13::rab-3::tdTomato, Podr-1::gfp] \\
\hline MTS735 & mig-1(e1787); wyls685 [Pmig-13::cla-1::gfp, Pmig-13:::rab-3::tdTomato, Podr-1::gfp] \\
\hline MTS716 & $\begin{array}{l}\text { mig-1(e1787); plr-1(shy20); wyls685 [Pmig-13::cla-1:::gfp, Pmig-13::rab- } \\
\text { 3::tdTomato, Podr-1::gfp] }\end{array}$ \\
\hline
\end{tabular}




\begin{tabular}{|c|c|}
\hline MTS963 & $\begin{array}{l}\text { mig-1(e1787); plr-1(shy20); wyls685 [Pmig-13::cla-1::gfp, Pmig-13:::rab- } \\
\text { 3::tdTomato, Podr-1::gfp]; shyEx249 [Pmig-13::mig-1, Podr-1::rfp] }\end{array}$ \\
\hline MTS964 & $\begin{array}{l}\text { mig-1(e1787); nrx-1(wy778), wyls685 [Pmig-13::cla-1::gfp, Pmig-13::rab- } \\
\text { 3::tdTomato, Podr-1::gfp] }\end{array}$ \\
\hline MTS561 & $\begin{array}{l}\text { lin-17(n3091); plr-1(shy20); wyls685 [Pmig-13::cla-1::gfp, Pmig-13::rab- } \\
\text { 3::tdTomato, Podr-1::gfp] }\end{array}$ \\
\hline MTS547 & lin-17(n3091); wyls685 [Pmig-13::cla-1::gfp, Pmig-13::rab-3::tdTomato, Podr-1::gfp] \\
\hline MTS337 & wyEx677 [Pmig-13:::lin-17::yfp + Pmig-13::mCherry::rab-3] \\
\hline MTS338 & plr-1(shy20); wyEx677 [Pmig-13::lin-17::yfp + Pmig-13::mCherry::rab-3] \\
\hline MTS234 & lin-17(n671); wyls685 [Pmig-13::cla-1::gfp, Pmig-13::rab-3::tdTomato, Podr-1::gfp] \\
\hline MTS965 & $\begin{array}{l}\text { plr-1(shy20); nrx-1(wy778), wyls685 [Pmig-13::cla-1::gfp, Pmig-13::rab- } \\
\text { 3::tdTomato, Podr-1::gfp] }\end{array}$ \\
\hline MTS471 & vab-8(shy8), wyls685 [Pmig-13::cla-1::gfp, Pmig-13::rab-3::tdTomato, Podr-1::gfp] \\
\hline MTS529 & $\begin{array}{l}\text { ptrn-1(js1286); vab-8(shy8), wyls685 [Pmig-13::cla-1::gfp, Pmig-13::rab- } \\
\text { 3::tdTomato, Podr-1::gfp] }\end{array}$ \\
\hline MTS955 & $\begin{array}{l}\text { vab-8(shy8), wyls685 [Pmig-13::cla-1::gfp, Pmig-13::rab-3::tdTomato, Podr-1::gfp]; } \\
\text { shyEx247 [Pmig-13::vab-8::scarlet, Punc-122::rfp] }\end{array}$ \\
\hline MTS966 & $\begin{array}{l}\text { vab-8(shy8), wyls685 [Pmig-13::cla-1:::gfp, Pmig-13::rab-3::tdTomato, Podr-1::gfp]; } \\
\text { shyEx250 [Pmig-13::vab-8(D1-326)::scarlet, Podr-1::rfp] }\end{array}$ \\
\hline MTS920 & $\begin{array}{l}\text { vab-8(shy8), wyls685 [Pmig-13::cla-1::gfp, Pmig-13::rab-3::tdTomato, Podr-1::gfp]; } \\
\text { shyEx235 [Pmig-13::vab-8(4713-1066)::scarlet, Podr-1::rfp] }\end{array}$ \\
\hline MTS923 & $\begin{array}{l}\text { vab-8(shy8), wyls685 [Pmig-13::cla-1:::gfp, Pmig-13::rab-3::tdTomato, Podr-1::gfp]; } \\
\text { shyEx236 [Pmig-13::vab-8(R278A):::scarlet, Podr-1::rfp] }\end{array}$ \\
\hline MTS976 & lin-44(n1792); wyls685 [Pmig-13::cla-1::gfp, Pmig-13::rab-3::tdTomato, Podr-1::gfp] \\
\hline MTS977 & $\begin{array}{l}\text { lin-44(n1792); vab-8(shy8), wyls685 [Pmig-13::cla-1::gfp, Pmig-13::rab- } \\
\text { 3::tdTomato, Podr-1::gfp] }\end{array}$ \\
\hline MTS662 & $\begin{array}{l}\text { lin-17(n3091); vab-8(shy8), wyls685 [Pmig-13::cla-1::gfp, Pmig-13::rab- } \\
\text { 3::tdTomato, Podr-1::gfp] }\end{array}$ \\
\hline MTS925 & $\begin{array}{l}\text { plr-1(shy20); vab-8(shy8), wyls685 [Pmig-13::cla-1::gfp, Pmig-13::rab-3::tdTomato, } \\
\text { Podr-1::gfp] }\end{array}$ \\
\hline MTS335 & vab-8(shy8); wyEx677 [Pmig-13::lin-17::yfp + Pmig-13::mCherry::rab-3] \\
\hline MTS729 & $\begin{array}{l}\text { mig-1(e1787); vab-8(shy8), wyls685 [Pmig-13::cla-1::gfp, Pmig-13::rab- } \\
\text { 3::tdTomato, Podr-1::gfp] }\end{array}$ \\
\hline MTS956 & vab-8(shy72), wyls685 [Pmig-13::cla-1::gfp, Pmig-13:::rab-3::tdTomato, Podr-1::gfp] \\
\hline MTS957 & $\begin{array}{l}\text { vab-8(shy73), nrx-1(wy778), wyls685 [Pmig-13::cla-1::gfp, Pmig-13::rab- } \\
\text { 3::tdTomato, Podr-1::gfp] }\end{array}$ \\
\hline MTS958 & $\begin{array}{l}\text { nrx-1(wy778), wyls685 [Pmig-13::cla-1::gfp, Pmig-13:::rab-3::tdTomato, Podr-1::gfp]; } \\
\text { shyEx248 [Pmig-13::vab-8, Punc-122::rfp] }\end{array}$ \\
\hline MTS538 & shyEx121 [Pitr-1::vab-8::gfp, Pmig-13::tagrfp, Pttx-3::rfp] \\
\hline MTS658 & shyEx175 [Pitr-1::vab-8::gfp, Pmig-13::rfp:::ptrn-1a, Punc-122::rfp] \\
\hline MTS967 & noca-1(ok3692); shyEx121 [Pitr-1::vab-8::gfp, Pmig-13::tagrfp, Pttx-3::rfp] \\
\hline MTS546 & plr-1(shy20); shyEx121 [Pitr-1::vab-8::gfp, Pmig-13::tagrfp, Pttx-3::rfp] \\
\hline MTS736 & $\begin{array}{l}\text { mig-1(e1787); plr-1(shy20); shyEx121 [Pitr-1::vab-8::gfp, Pmig-13::tagrfp, Pttx- } \\
\text { 3::rfp] }\end{array}$ \\
\hline MTS968 & nrx-1(wy778); shyEx121 [Pitr-1::vab-8::gfp, Pmig-13::tagrfp, Pttx-3::rfp] \\
\hline MTS969 & $\begin{array}{l}\text { mig-1(e1787); nrx-1(wy778); shyEx121 [Pitr-1::vab-8::gfp, Pmig-13::tagrfp, Pttx- } \\
\text { 3::rfp] }\end{array}$ \\
\hline MTS970 & $\begin{array}{l}\text { plr-1(shy20); nrx-1(wy778); shyEx121 [Pitr-1::vab-8::gfp, Pmig-13::tagrfp, Pttx- } \\
\text { 3::rfp] }\end{array}$ \\
\hline
\end{tabular}




\begin{tabular}{|c|c|}
\hline MTS226 & $\begin{array}{l}\text { tba-1(ok1135); wySi265 [Punc-4c:::CRE; Punc-122::rfp]; wyls463 [Pmig- } \\
\text { 13::STOP::gfp::tba-1; Podr-1::rfp] }\end{array}$ \\
\hline MTS516 & $\begin{array}{l}\text { tba-1(ok1135); vab-8(shy8); wySi265 [Punc-4c::CRE; Punc-122::rfp]; wyls463 } \\
\text { [Pmig-13::STOP::gfp::tba-1; Podr-1::rfp] }\end{array}$ \\
\hline MTS427 & $\begin{array}{l}\text { tba-1(ok1135); plr-1(shy20); wySi265 [Punc-4c::CRE; Punc-122::rfp]; wyls463 } \\
\text { [Pmig-13::STOP::gfp::tba-1; Podr-1::rfp] }\end{array}$ \\
\hline TV21554 & wyls802 [Pitr-1::yfp::ptrn-1a, Podr-1::gfp] \\
\hline MTS126 & vab-8(shy8); wyls802 [Pitr-1::yfp::ptrn-1a, Podr-1::gfp] \\
\hline MTS998 & nrx-1(wy778); wyls802 [Pitr-1::yfp:::ptrn-1a, Podr-1::gfp] \\
\hline MTS717 & mig-1(e1787); vab-8(shy8); wyls802 [Pitr-1::yfp::ptrn-1a, Podr-1::gfp] \\
\hline MTS310 & vab-8(ev411); wyls802 [Pitr-1::yfp::ptrn-1a, Podr-1::gfp] \\
\hline MTS284 & vab-8(e1017); wyls802 [Pitr-1::yfp::ptrn-1a, Podr-1::gfp] \\
\hline MTS431 & plr-1(shy20); vab-8(shy8); wyls802 [Pitr-1::yfp:::ptrn-1a, Podr-1::gfp] \\
\hline MTS285 & vab-8(gm84); wyls802 [Pitr-1::yfp::ptrn-1a, Podr-1::gfp] \\
\hline MTS465 & unc-104(e1265); vab-8(shy8); wyls803 [Pitr-1::yfp:::ptrn-1a, Podr-1::gfp] \\
\hline MTS534 & tbb-2(qt1); vab-8(shy8); wyls802 [Pitr-1::yfp::ptrn-1a, Podr-1::gfp] \\
\hline MTS979 & $\begin{array}{l}\text { tbb-2(qt1); vab-8(shy8); wyls685 [Pmig-13::cla-1::gfp, Pmig-13::rab-3::tdTomato, } \\
\text { Podr-1::gfp] }\end{array}$ \\
\hline MTS978 & unc-104(e1265); wyls803 [Pitr-1::yfp::ptrn-1a, Podr-1::gfp] \\
\hline MTS980 & tbb-2(qt1); wyls685 [Pmig-13::cla-1::gfp, Pmig-13::rab-3::tdTomato, Podr-1::gfp] \\
\hline MTS981 & tbb-2(qt1); wyls802 [Pitr-1::yfp::ptrn-1a, Podr-1::gfp] \\
\hline MTS226 & $\begin{array}{l}\text { tba-1(ok1135); wySi265 [Punc-4c::CRE; Punc-122::rfp]; wyls463 [Pmig- } \\
\text { 13::STOP::gfp::tba-1; Podr-1::rfp]; wyEx7468 [Pmig-13::STOP::tagrfp; Podr-1::gfp] }\end{array}$ \\
\hline MTS613 & $\begin{array}{l}\text { tba-1(ok1135); vab-8(shy8); wySi265 [Punc-4c::CRE; Punc-122::rfp]; wyls463 } \\
\text { [Pmig-13::STOP::gfp::tba-1; Podr-1::rfp]; wyEx7468 [Pmig-13::STOP::tagrfp; Podr- } \\
\text { 1::gfp] }\end{array}$ \\
\hline MTS428 & $\begin{array}{l}\text { tba-1(ok1135); plr-1(shy20); wySi265 [Punc-4c::CRE; Punc-122::rfp]; wyls463 } \\
\text { [Pmig-13::STOP::gfp::tba-1; Podr-1::rfp]; wyEx7468 [Pmig-13::STOP::tagrfp; Podr- } \\
\text { 1::gfp] }\end{array}$ \\
\hline MTS482 & $\begin{array}{l}\text { vab-8(shy8); wyls802 [Pitr-1::yfp:::ptrn-1a, Podr-1::gfp]; shyEx247 [Pmig-13::vab- } \\
\text { 8::scarlet, Punc-122:::ff]] }\end{array}$ \\
\hline MTS479 & $\begin{array}{l}\text { vab-8(shy8); wyls802 [Pitr-1::yfp:::ptrn-1a, Podr-1::gfp]; shyEx250 [Pmig-13::vab- } \\
8(\Delta 1-326):: \text { scarlet, Podr-1::rfp] }\end{array}$ \\
\hline MTS990 & 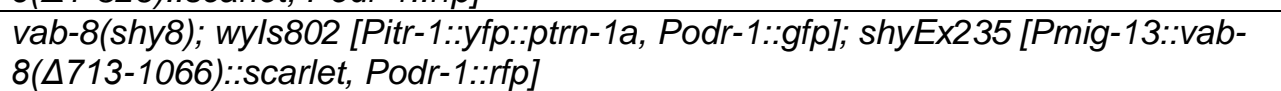 \\
\hline MTS991 & $\begin{array}{l}\text { vab-8(shy8); wyls802 [Pitr-1::yfp::ptrn-1a, Podr-1::gfp]; shyEx236 [Pmig-13::vab- } \\
8(\text { R278A)::scarlet, Podr-1::rfp] }\end{array}$ \\
\hline MTS92 & plr-1(shy20); wyls802 [Pitr-1::yfp::ptrn-1a, Podr-1::gfp] \\
\hline MTS340 & plr-1(hd129); wyls802 [Pitr-1::yfp:::ptrn-1a, Podr-1::gfp] \\
\hline MTS637 & mig-1(e1787); plr-1(shy20); wyls802 [Pitr-1::yfp::ptrn-1a, Podr-1::gfp] \\
\hline MTS992 & $\begin{array}{l}\text { plr-1(shy20); wyls802 [Pitr-1::yfp:::ptrn-1a, Podr-1::gfp]; shyEx241 [Pmig-13::plr- } \\
\text { 1::scarlet, Punc-122::rfp] }\end{array}$ \\
\hline MTS993 & $\begin{array}{l}\text { plr-1(shy20); wyls802 [Pitr-1::yfp::ptrn-1a, Podr-1::gfp]; shyEx234 [Pmig-13::plr- } \\
\text { 1((4317-358::scarlet), Punc-122:::fp] }\end{array}$ \\
\hline MTS510 & $\begin{array}{l}\text { wyls802 [Pitr-1::yfp:::ptrn-1a, Podr-1::gfp], wySi265 [Punc-4c:::CRE; Punc-122::rfp]; } \\
\text { wyls363 [Pmig-13::STOP::tdTomato::rab-3; Podr-1::gfp] }\end{array}$ \\
\hline MTS609 & $\begin{array}{l}\text { wyls802 [Pitr-1::yfp:::ptrn-1a, Podr-1::gfp], wySi265 [Punc-4c:::CRE; Punc-122::rfp]; } \\
\text { vab-8(shy8); wyls363 [Pmig-13::STOP::tdTomato::rab-3; Podr-1::gfp] }\end{array}$ \\
\hline
\end{tabular}




\begin{tabular}{|c|c|}
\hline MTS661 & $\begin{array}{l}\text { wyls802 [Pitr-1::yfp:::ptrn-1a, Podr-1::gfp], wySi265 [Punc-4c::CRE; Punc-122::rfp]; } \\
\text { plr-1(shy20); wyls363 [Pmig-13::STOP::tdTomato::rab-3; Podr-1::gfp] }\end{array}$ \\
\hline MTS994 & shyEx251[Pitr-1::noca-1b::gfp, Podr-1::rfp] \\
\hline MTS995 & vab-8(shy8); shyEx251[Pitr-1::noca-1b::gfp, Podr-1::rfp] \\
\hline MTS996 & plr-1(shy20); shyEx251[Pitr-1::noca-1b::gfp, Podr-1::rfp] \\
\hline TV9315 & wyls251 [Pmig-13::pB::gfp:::rab-3, Podr-1::gfp] \\
\hline MTS535 & vab-8(shy8); wyls251 [Pmig-13::rab-3::gfp, Podr-1::gfp] \\
\hline MTS463 & plr-1(shy20); wyls251 [Pmig-13::pB:::gfp:::rab-3, Podr-1::gfp] \\
\hline MTS774 & $\begin{array}{l}\text { dhc-I(js319); vab-8(shy8), wyls685 [Pmig-13::cla-1::gfp, Pmig-13::rab-3::tdTomato, } \\
\text { Podr-1::gfp] }\end{array}$ \\
\hline MTS773 & $\begin{array}{l}\text { nud-2(ok949); vab-8(shy8), wyls685 [Pmig-13::cla-1::gfp, Pmig-13::rab- } \\
\text { 3::tdTomato, Podr-1::gfp] }\end{array}$ \\
\hline MTS986 & $\begin{array}{l}\text { unc-104(wy711); vab-8(shy8), wyls685 [Pmig-13::cla-1::gfp, Pmig-13::rab- } \\
\text { 3::tdTomato, Podr-1::gfp] }\end{array}$ \\
\hline MTS987 & $\begin{array}{l}\text { arl-8(tm2388); vab-8(shy8), wyls685 [Pmig-13::cla-1::gfp, Pmig-13::rab- } \\
\text { 3::tdTomato, Podr-1::gfp] }\end{array}$ \\
\hline MTS983 & dhc-I(js319); wyls685 [Pmig-13::cla-1::gfp, Pmig-13:::rab-3::tdTomato, Podr-1::gfp] \\
\hline MTS982 & nud-2(ok949); wyls685 [Pmig-13::cla-1::gfp, Pmig-13::rab-3::tdTomato, Podr-1::gfp] \\
\hline MTS988 & $\begin{array}{l}\text { unc-104(wy711); wyls685 [Pmig-13::cla-1::gfp, Pmig-13::rab-3::tdTomato, Podr- } \\
1:: \text { gfp] }\end{array}$ \\
\hline MTS989 & arl-8(tm2388); wyls685 [Pmig-13::cla-1::gfp, Pmig-13::rab-3::tdTomato, Podr-1::gfp] \\
\hline MTS985 & $\begin{array}{l}\text { dhc-l(js319); plr-1(shy20); wyls685 [Pmig-13::cla-1::gfp, Pmig-13::rab-3::tdTomato, } \\
\text { Podr-1::gfp] }\end{array}$ \\
\hline MTS984 & $\begin{array}{l}\text { dhc-l(js319); nrx-1(wy778); wyls685 [Pmig-13::cla-1::gfp, Pmig-13::rab- } \\
\text { 3::tdTomato, Podr-1::gfp] }\end{array}$ \\
\hline
\end{tabular}

Table S1: Description of alleles used

\begin{tabular}{|c|c|c|}
\hline Allele & Molecular change & Effect on protein/function \\
\hline vab-8(shy8) & $\begin{array}{l}\text { Deletion and frameshift: } \\
\text { 463KLYDELC to } \\
\text { 463TDDKKR-Opal }\end{array}$ & $\begin{array}{l}\text { Truncation of the protein before coiled-coil domain } \\
\text { (CCD) (see Figure } 2 F \text { ) / null allele (see Figure S5) }\end{array}$ \\
\hline$v a b-8($ shy 72$)$ & CRISPR insertion R452Ochre & $\begin{array}{l}\text { Truncation of the protein before CCD (see Figure } \\
2 \mathrm{~F} \text { and Methods) }\end{array}$ \\
\hline$v a b-8(e v 411)$ & $\begin{array}{l}\text { Substitution - Splice donor } \\
\text { (G904A) }\end{array}$ & Truncation of the protein in motor domain (MD) \\
\hline$v a b-8(e 1017)$ & $\begin{array}{l}\text { Substitution - Stop codon } \\
\text { (R554Opal) }\end{array}$ & Truncation of the protein in CCD \\
\hline$v a b-8(g m 84)$ & $\begin{array}{l}\text { Substitution - Splice donor } \\
\text { (G10,698A) }\end{array}$ & Truncation of the protein in CCD \\
\hline plr-1(shy20) & $\begin{array}{l}\text { Substitution - Stop codon } \\
\text { (R1880pal) }\end{array}$ & $\begin{array}{l}\text { Truncation of the protein before transmembrane } \\
\text { domain (TMD) (see Figure 1C). }\end{array}$ \\
\hline plr-1(hd129) & Deletion & Truncation of the protein before TMD \\
\hline $\operatorname{lin}-17(n 3091)$ & $\begin{array}{l}\text { Substitution - Stop codon } \\
\text { (Q55Ochre) }\end{array}$ & Early truncation (before TM domains) \\
\hline $\operatorname{lin}-17(n 671)$ & $\begin{array}{l}\text { Substitution - Stop codon } \\
\text { (Q449Ochre) }\end{array}$ & Truncation of the protein after 6th TMD \\
\hline $\operatorname{mig}-1(e 1787)$ & $\begin{array}{l}\text { Q277Ochre (transcript A) or } \\
\text { Q281Ochre (transcript B) }\end{array}$ & Truncation between 3rd and 4th TM domains \\
\hline
\end{tabular}




\begin{tabular}{|c|c|c|}
\hline$n r x-1($ wy 778$)$ & Deletion & $\begin{array}{l}\text { Deletion covering } \mathrm{C} \text { terminus of } \alpha \text { and } \gamma \mathrm{nrx}-1 \\
\text { isoforms (including transmembrane and } \\
\text { intracellular domain) }\end{array}$ \\
\hline lin-44(n1792) & $\begin{array}{l}\text { Substitution - Stop codon } \\
\text { (W100Amber) }\end{array}$ & Truncation of the protein in WNT-1 domain \\
\hline dhc-I(js319) & $\begin{array}{l}\text { Substitution - Splice acceptor } \\
\text { intron } 12 \text { (Cter) }\end{array}$ & $\begin{array}{l}\text { Weak allele based on viability and comparison to } \\
\text { other alleles (Koushika et al., 2004, Yogev et al., } \\
\text { 2017) }\end{array}$ \\
\hline nud-2(ok949) & Deletion & $\begin{array}{l}\text { Truncation of the globular domain and CCD. Does } \\
\text { not severly affect SVP transport (Ou et al., 2010) }\end{array}$ \\
\hline $\begin{array}{l}\text { unc- } \\
104(\text { wy } 711)\end{array}$ & Substitution - G313E & Weak allele (Wu et al, 2013) \\
\hline $\begin{array}{l}\text { unc- } \\
104(e 1265)\end{array}$ & Substitution - D1497N & $\begin{array}{l}\text { Disruption of } \mathrm{PH} \text { domain, strong loss of function } \\
\text { but not null. }\end{array}$ \\
\hline arl-8(tm2388) & Deletion in 3'UTR & Weak allele (Wu et al., 2013) \\
\hline$t b b-2(q t 1)$ & Substitution - E198K & $\begin{array}{l}\text { Temperature sensitive, attenuates microtubule } \\
\text { dynamics in DA9 (Yogev et al., 2017) }\end{array}$ \\
\hline $\begin{array}{l}\text { noca- } \\
1 \text { (ok3692) }\end{array}$ & Deletion & $\begin{array}{l}\text { Removes ninein homology domain (NHD), } \\
\text { homozygous sterile. }\end{array}$ \\
\hline $\begin{array}{l}\text { ptrn- } \\
1(j s 1286)\end{array}$ & $\begin{array}{l}\text { Substitution - Stop codon } \\
\text { (Y241Amber) }\end{array}$ & $\begin{array}{l}\text { Truncation of the protein in calponin-homology } \\
(\mathrm{CH}) \text { domain }\end{array}$ \\
\hline
\end{tabular}

\section{Acknowledgments}

The authors would like to thank the Caenorhabditis Genetic Center (CGC) for strains. We also thank Kang Shen for sharing strains and D. Miller for the Prab-3::spGFP ${ }_{1-10}$ strain. We thank members of the Yogev and Hammarlund labs for discussions and technical assistance. This work was supported by NIH R01-NS114400 and R35-GM131744.

\section{Author contributions}

S.B.G and S.Y designed the project, carried out experiments in C. elegans and wrote the manuscript. L.S.: Matlab codes for image analysis. S.C and C.K: cloning and imaging. Y.Y and K.J.V: design, imaging and interpretation of in vitro experiments. 


\section{Figure S1}

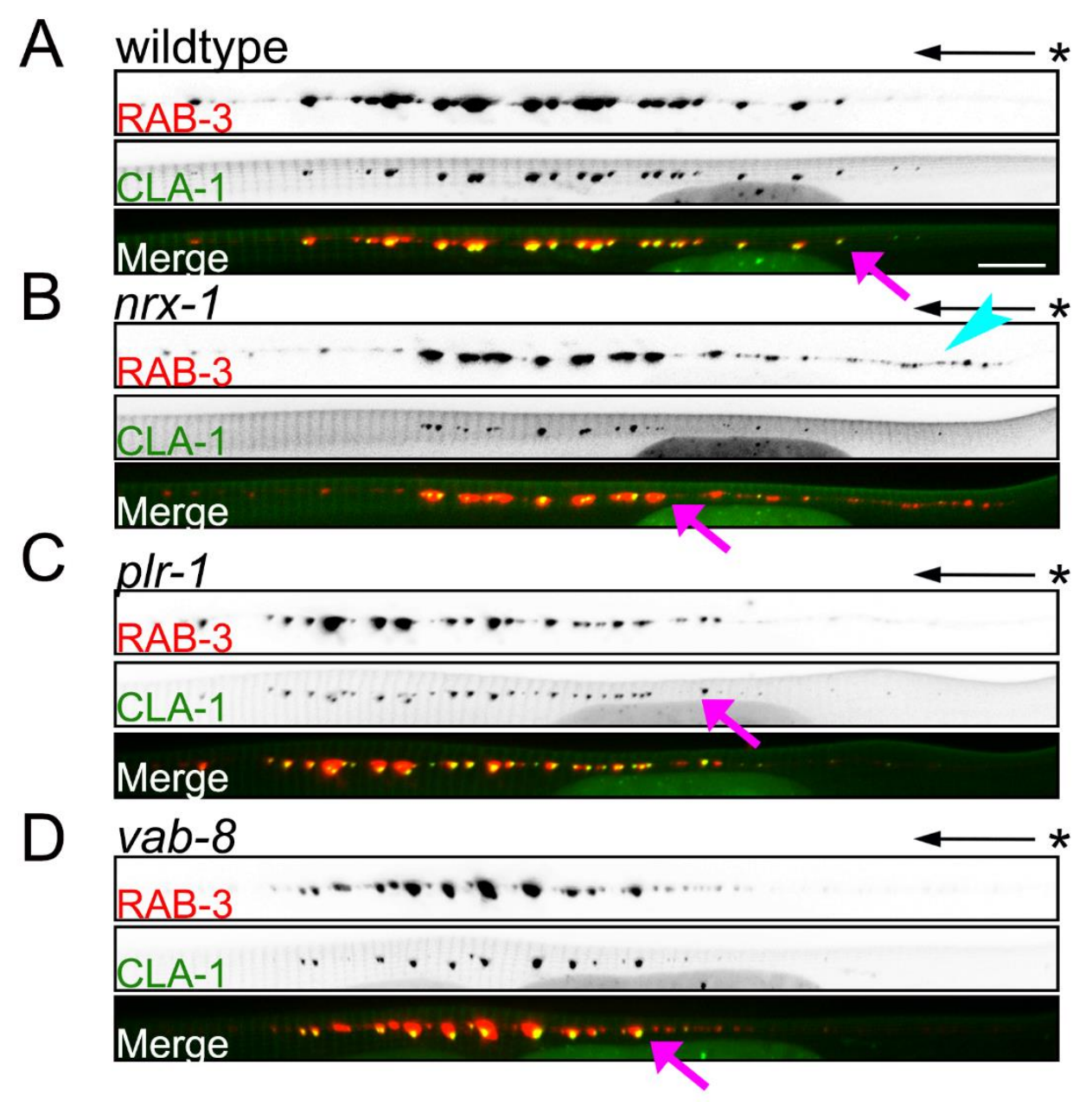

Figure S1: Normal synaptic vesicle clustering in vab-8 and plr-1 mutants

(A-D) vab-8 and plr-1 mutants phenocopy the missing proximal synapses of $n r x-1$ but do not show vesicle clustering defects. Axons from wildtype (A), $n r x-1$ (B), plr-1 (C) and vab-8 (D) expressing the active zone marker GFP::CLA-1 and the synaptic vesicle precursor marker tdTomato::RAB-3. * marks the turn of the commissure. Pink arrows point to the first synapse and arrowhead points to RAB- 3 that is not associated with CLA in $n r x-1$ mutants, indicating vesicle clustering defects. Scale bar $=5 \mu \mathrm{m}$. 


\section{Figure S2}

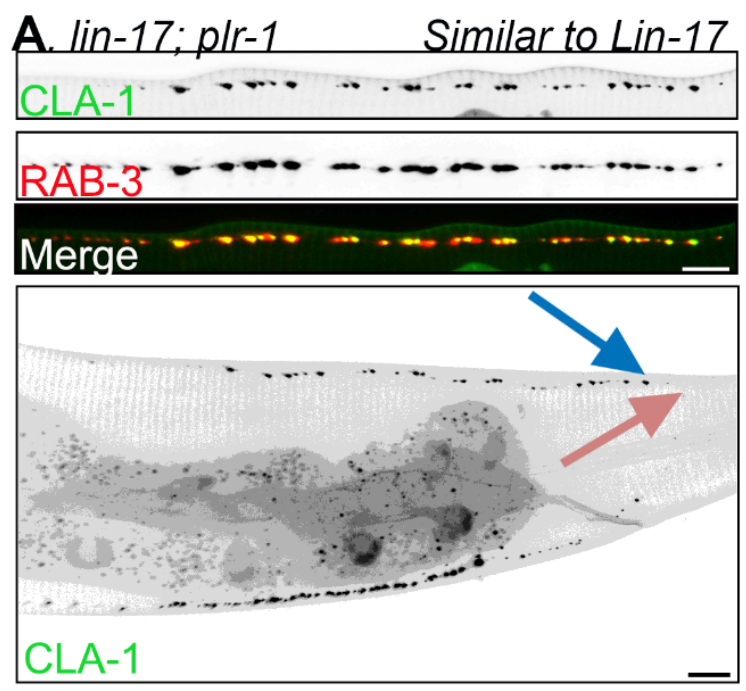

C. lin-17; plr-1 intermediate phenotype

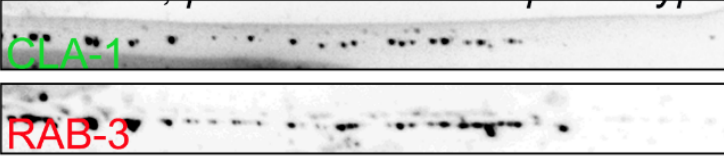

Merge ….............

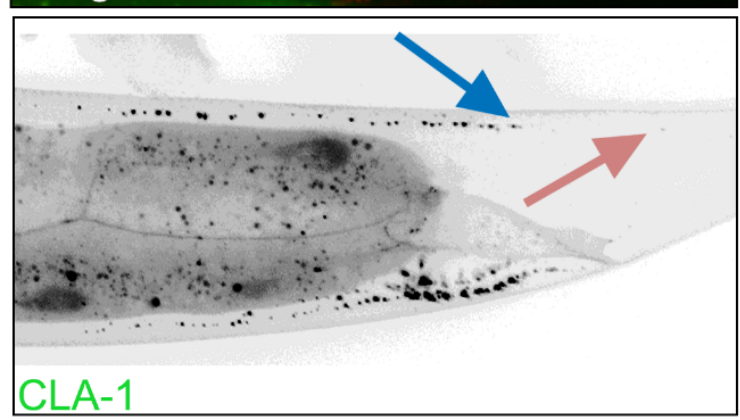

\section{E wildtype}

LIN 17 / IFZ

RAB-3

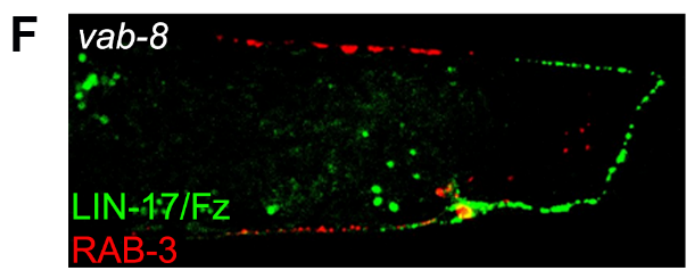

B. lin-17; $p / r-1$

Similar to Plr-1 CLA-1

RAB-3

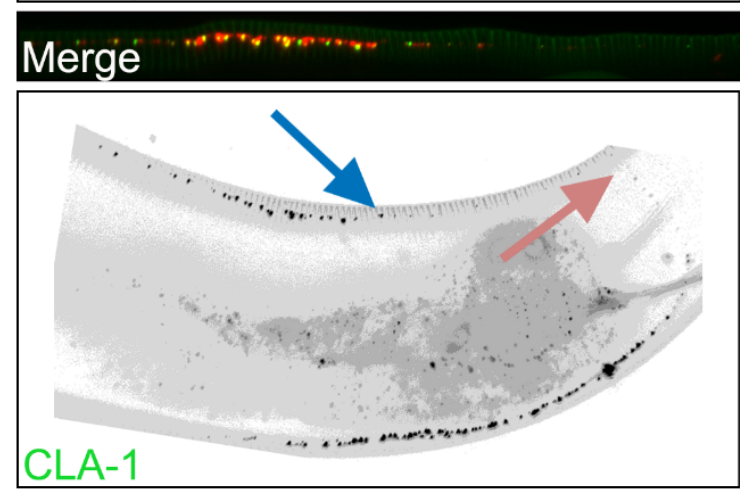

D Similar to Lin-17

Intermediate phenotype

Similar to P/r-1
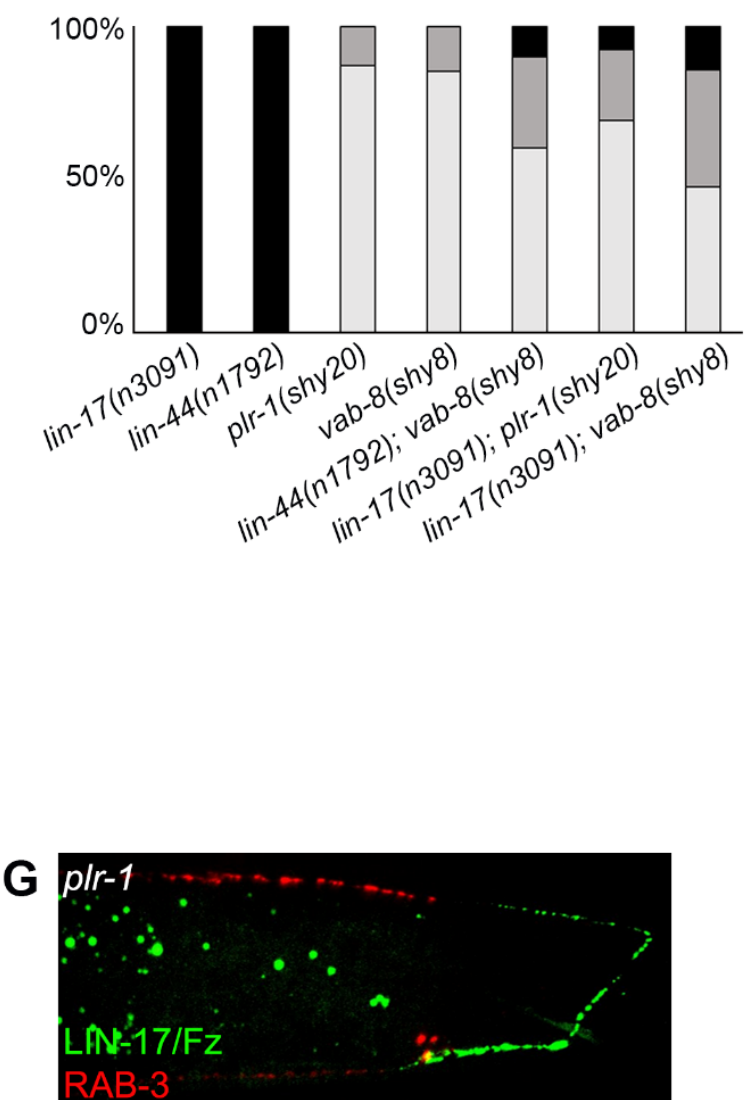


\section{Figure S2: The Frizzled receptor LIN-17 does not act downstream of PLR-1}

(A-C) Representative images of the different phenotypic classes used for quantifying genetic interactions. All images are from lin-17; plr-1 double mutants expressing the active zone marker GFP::CLA-1 and the synaptic vesicle precursor marker tdTomato::RAB-3. Pink arrow marks the turn of the commissure and blue arrow marks the first synapse. "Lin 17 phenotype" is characterized by synapses starting immediately following the turn of the commissure or in the commissure itself. "Plr-1 phenotype" is defined as an asynaptic domain that is larger than in wildtype. "intermediate phenotype" is characterized by synapses starting at their normal location of closer to the turn of the commissure. (D) Quantification of the fraction of each phenotypic class in the indicated mutant backgrounds. (E-G) Representative images of wildtype (E), vab-8 (F) and plr-1 (G) adult worms expressing Frizzled/LIN17::YFP and mCherry::RAB-3 in DA9. No apparent changes in the localization of Frizzled/LIN-17 is observed in the mutants. Scale bar $=5 \mu \mathrm{m}$. 


\section{Figure S3}

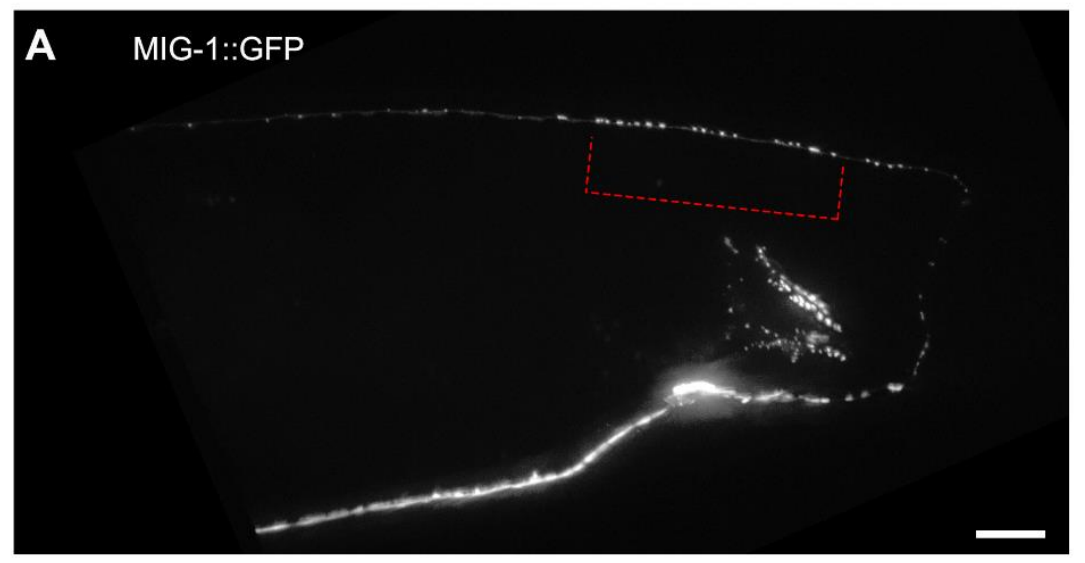

Figure S3: Axonal Frizzled/MIG-1 is localized to the region that shows synaptic defects in p/r1 mutants

Image shows adult worm expressing MIG-1::GFP in DA9. Red brackets indicate the location where synapses are missing in plr-1 mutants. Scale bar $=5 \mu \mathrm{m}$. 


\section{Figure S4}

A

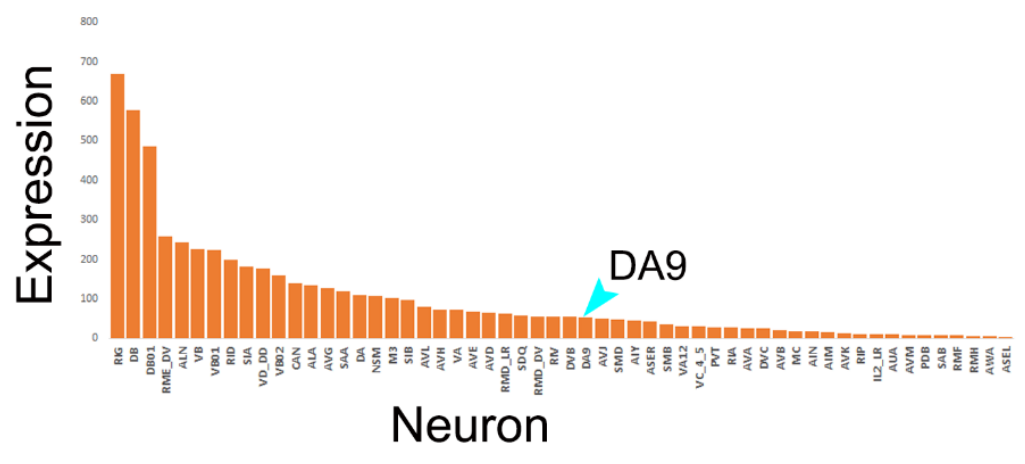

B

ALN/SDQL

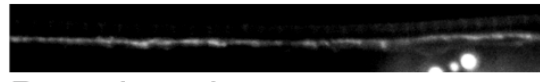

Dorsal cord

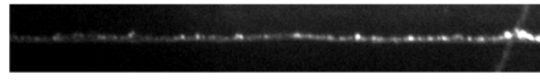

SIAV/SIBV/SMBV

Figure S4: Endogenous VAB-8 expression and subcellular distribution

(A) Expression level of VAB-8 in single-cell RNAseq experiments from the CenGen project:

https://cengen.shinyapps.io/CengenApp/. DA9 is indicated. (B) select examples of neurons where VAB-8 expression was sufficiently high to be detected with an endogenous $7 X_{s p G F P}$ tag and pan-neuronal expression of spGFP 1 -10. Neuron identity was assigned based on the position of the axon (in most cases cell-bodies were too dim) and therefore contains some ambiguity. 


\section{Figure S5}

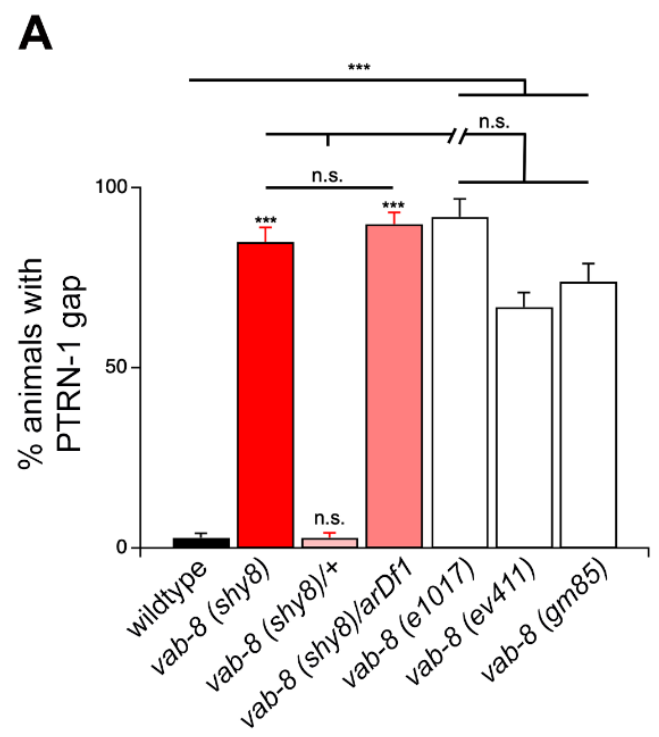

B

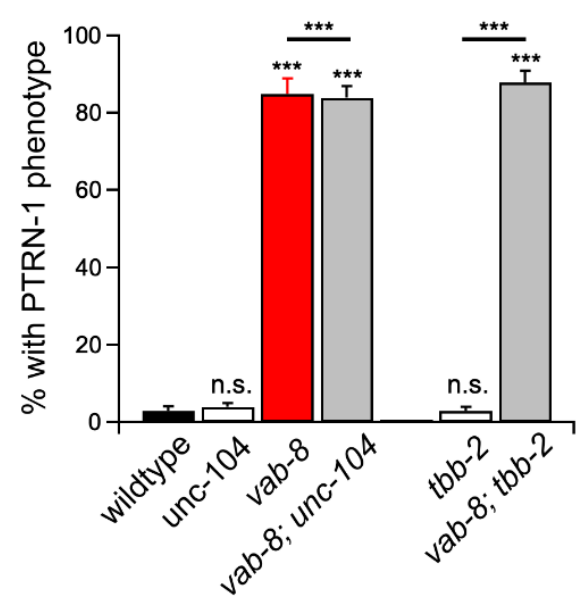

C

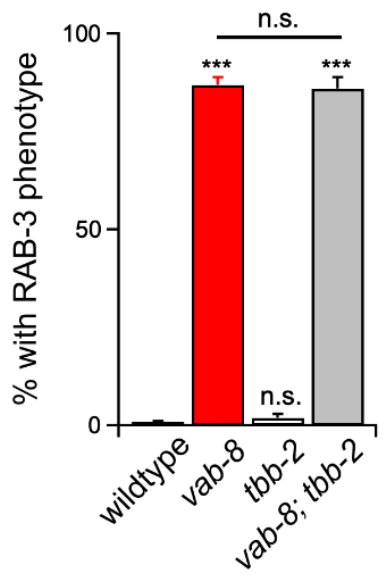

Figure S5: PTRN-1 gaps in vab-8 mutants are not caused by the loss of synapses and loss of microtubule dynamicity does not lead to synapse elimination

(A) Comparison to previously characterized alleles and a deletion of the vab-8 region shows that shy 8 is a genetic null allele. The presence of a PTRN-1::YFP "gap" around proximal synapses was scored.

(B) The presence of PTRN-1 defects was tested in unc-104(e1265) in which DA9 synapses are largely eliminated and in tbb-2(qt1) mutants in which microtubule dynamicity is strongly suppressed in DA9. In both cases, no PTRN-1::YFP "gaps" are seen, and double mutants with vab-8 do not modify the $V a b-8$ phenotype, suggesting that loss of synapses and reduced microtubule dynamics do not drive the loss of PTRN-1::YFP in vab-8 mutants. (C) Suppressing microtubule dynamics in tbb-2(qt1) mutants does not lead to loss of proximal synapses and does not modify the loss of proximal synapses in vab-8 mutants. $\mathrm{n}=100-150$ per genotype, ${ }^{* * *} p<0.001$; (Mann-Whitney $U$ test). 


\section{Figure S6}

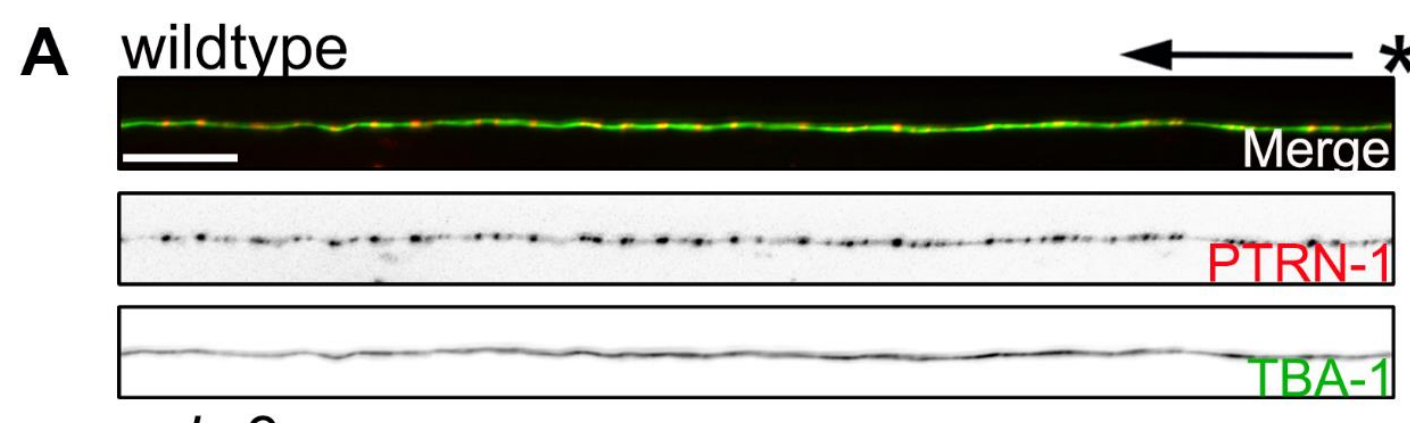

B $v a b-8$
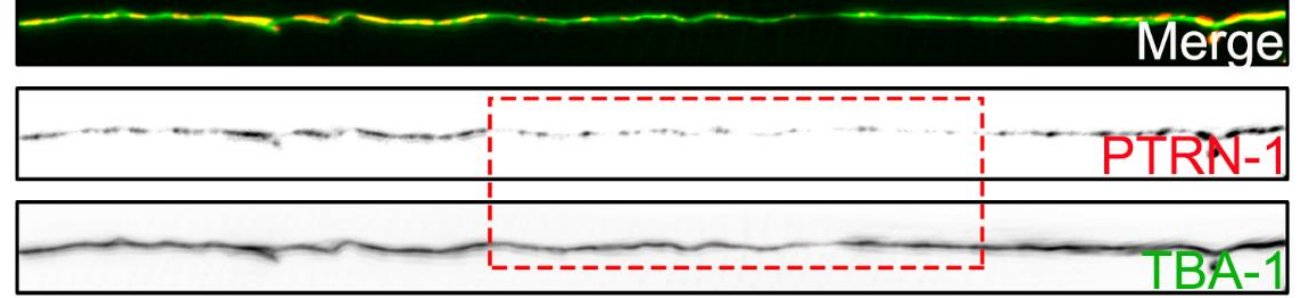

C $p / r-1$

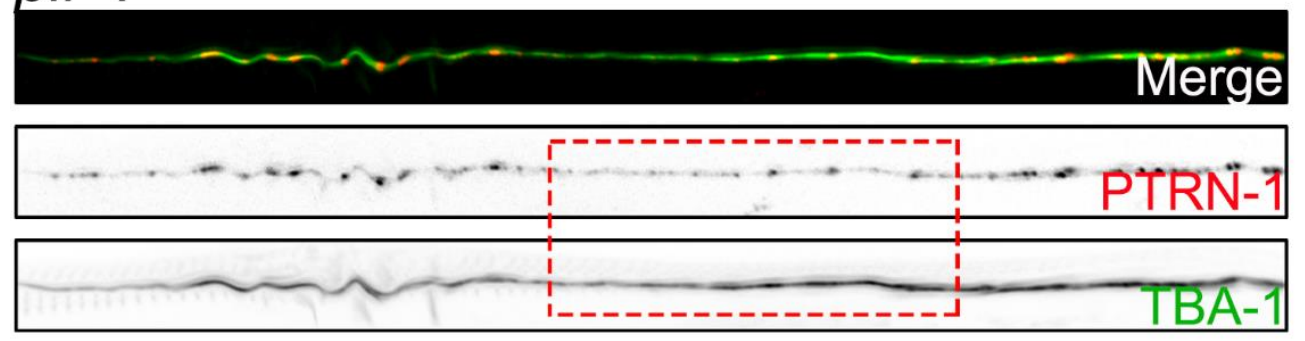

Figure S6: Microtubules are present in the region that shows PTRN-1 gaps in vab-8 and p/r-1 mutants

(A-C) Images of axons from wildtype (A), $v a b-8$ (B) and plr-1 (C) mutants expressing RFP::PTRN-1 and GFP::TBA-1/ $\alpha$-tubulin. Loss of PTRN-1 is not associated with loss of microtubules. * marks turn of the commissure. Dashed boxes highlight the affected region. Scale bar $=5 \mu \mathrm{m}$. 


\section{Figure S6}

A

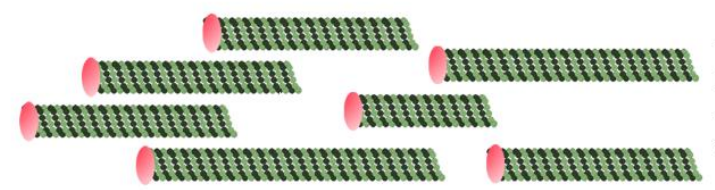

- Average GFP::TBA-1/ $\alpha$-tubulin intensity around all RF-

P.:PTRN-1 peaks ("on") or halfway in between ("off").

- If PTRN-1 puncta localize to MT tips, average GFP should near a peak at PTRN-1 signal.

B

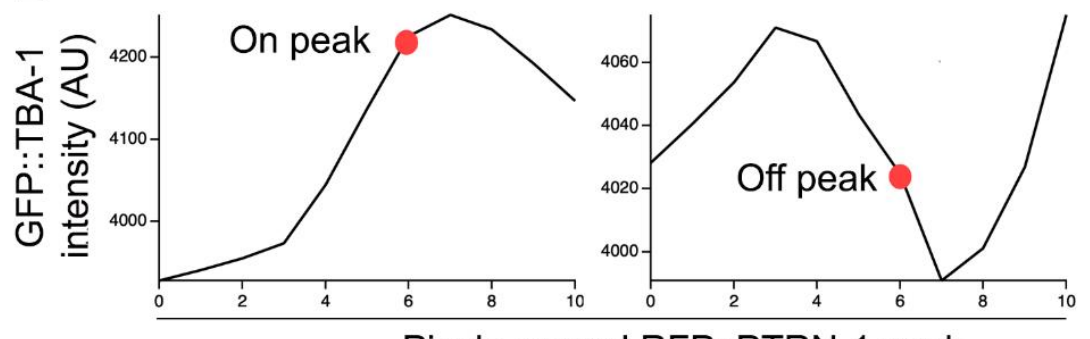

Pixels around RFP::PTRN-1 peak

C

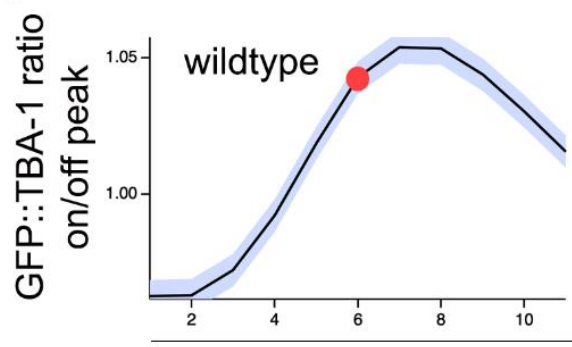

G

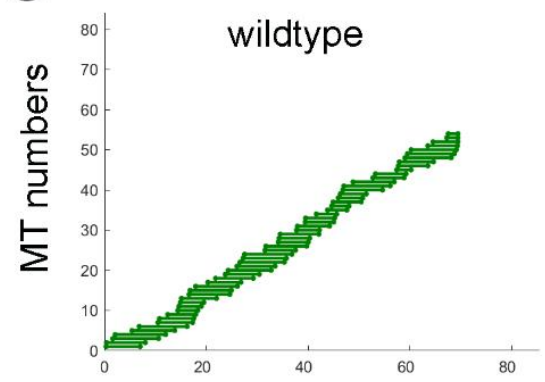

D

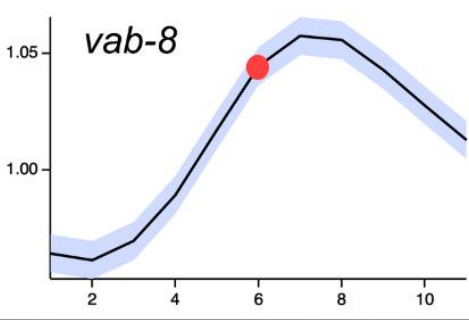

\section{H}

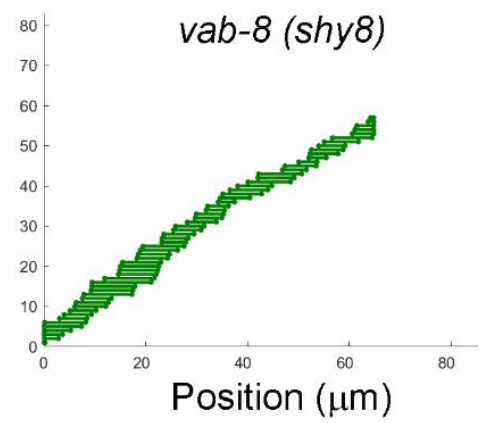

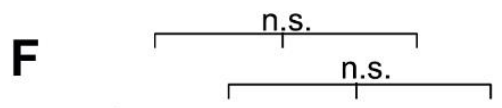

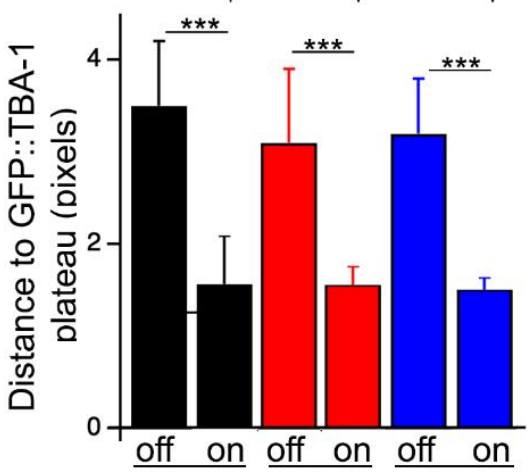

E

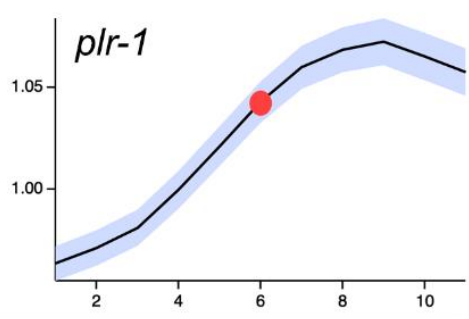

Pixels around RFP::PTRN-1 peak

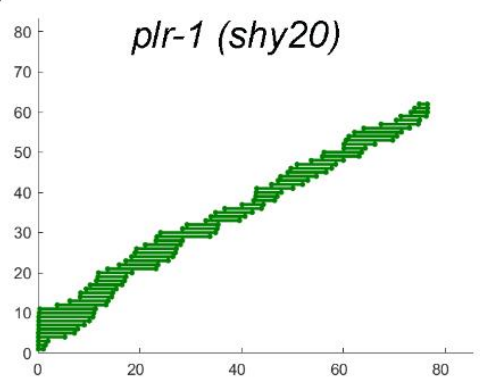

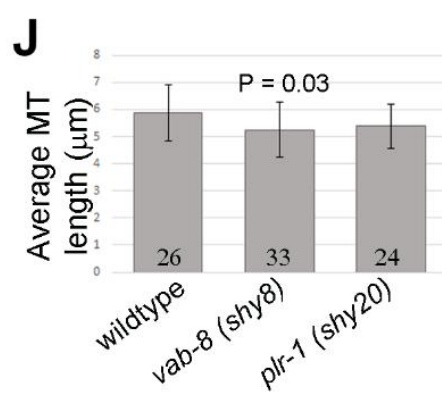

K :

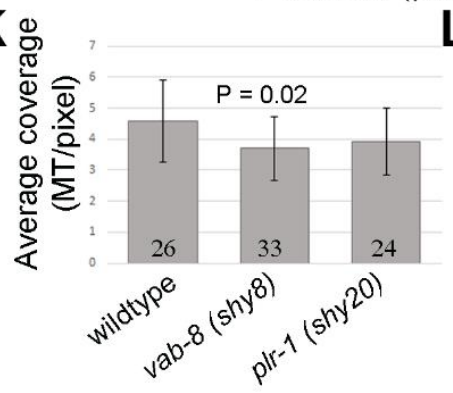

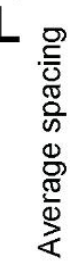

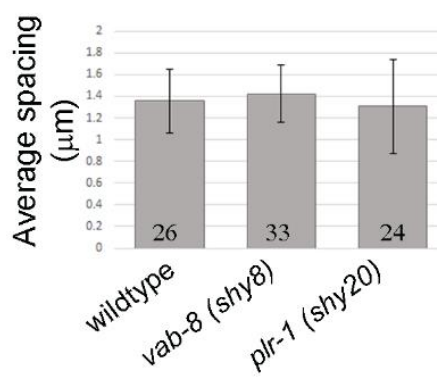


Figure S7: Normal minus-end localization of PTRN-1 and normal microtubule organization in p/r-1 and vab-8 mutants.

(A) Schematic of the concept for determining association of RFP::PTRN-1 with microtubule ends. The intensity of GFP::TBA-1 from an individual microtubule that harbors a given RFP::PTRN-1 punctum cannot be distinguished from that of other microtubules near it and so it is not possible to test where individual PTRN-1 puncta are localized along a given polymer. However, the average GFP::TBA-1 around many RFP::PTRN-1 puncta should increase and near a plateau is the puncta are associated with microtubule ends. (B) Example from a single wildtype animal: average GFP::TBA-1/ $\alpha$-tubulin around all RFP::PTRN-1 puncta from a $45 \mu \mathrm{m}$ region around proximal synapses shows the expected increase. As a negative control, GFP::TBA-1/ $\alpha$-tubulin shows a decrease around the halfway point between PTRN-1 puncta. (C-E) Identical behavior of GFP::TBA-1/ $\alpha$-tubulin around RFP::PTRN-1 peaks in wildtype (C), $v a b-8$ (D) and plr-1 (E) confirms that the faint RFP::PTRN-1 puncta in the mutants are associated with microtubule minus-ends. (F) Quantification of the distance between PTRN-1 puncta ("on") and the halfway between them ("off") to the nearest peak in GFP::TBA-1/ $\alpha$-tubulin intensity shows no difference between wildtype and mutants. (G-L) wildtype animals (G, J), vab-8 mutants $(\mathrm{H}, \mathrm{K})$ and plr-1 mutants $(\mathrm{I}, \mathrm{L})$ show overall similar microtubule organization. Microtubule organization parameters were determined as described in (Yogev et al., 2016). (G,H,I) show models for individual animals. (J) Average polymer length. (K) Average number of polymers per "cross section" of the axon at a given pixel. (L) Spacing between microtubule ends. $n=20-25$. 


\section{$\underline{\text { References }}$}

Ahmari, S.E., Buchanan, J., and Smith, S.J. (2000). Assembly of presynaptic active zones from cytoplasmic transport packets. Nat Neurosci 3, 445-451.

Akhmanova, A., and Steinmetz, M.O. (2019). Microtubule minus-end regulation at a glance. J Cell Sci 132.

Bharat, V., Siebrecht, M., Burk, K., Ahmed, S., Reissner, C., Kohansal-Nodehi, M., Steubler, V., Zweckstetter, M., Ting, J.T., and Dean, C. (2017). Capture of Dense Core Vesicles at Synapses by JNKDependent Phosphorylation of Synaptotagmin-4. Cell Rep 21, 2118-2133.

Biederer, T., and Südhof, T.C. (2000). Mints as adaptors. Direct binding to neurexins and recruitment of munc18. J Biol Chem 275, 39803-39806.

Biederer, T., and Sudhof, T.C. (2001). CASK and protein 4.1 support F-actin nucleation on neurexins. J Biol Chem 276, 47869-47876.

Brenner, S. (1974). The genetics of Caenorhabditis elegans. Genetics 77, 71-94.

Brouwer, M., Farzana, F., Koopmans, F., Chen, N., Brunner, J.W., Oldani, S., Li, K.W., van Weering, J.R., Smit, A.B., Toonen, R.F., et al. (2019). SALM1 controls synapse development by promoting F-actin/PIP2dependent Neurexin clustering. EMBO J 38, e101289.

Cai, D., McEwen, D.P., Martens, J.R., Meyhofer, E., and Verhey, K.J. (2009). Single molecule imaging reveals differences in microtubule track selection between Kinesin motors. PLoS Biology 7, e1000216.

Cavolo, S.L., Bulgari, D., Deitcher, D.L., and Levitan, E.S. (2016). Activity Induces Fmr1-Sensitive Synaptic Capture of Anterograde Circulating Neuropeptide Vesicles. J Neurosci 36, 11781-11787.

Chen, X., Shibata, A.C., Hendi, A., Kurashina, M., Fortes, E., Weilinger, N.L., MacVicar, B.A., Murakoshi, H., and Mizumoto, K. (2018). Rap2 and TNIK control Plexin-dependent tiled synaptic innervation in C. elegans. ELife 7, e38801.

Dean, C., Scholl, F.G., Choih, J., DeMaria, S., Berger, J., Isacoff, E., and Scheiffele, P. (2003). Neurexin mediates the assembly of presynaptic terminals. Nat Neurosci $6,708-716$.

Dokshin, G.A., Ghanta, K.S., Piscopo, K.M., and Mello, C.C. (2018). Robust Genome Editing with Short Single-Stranded and Long, Partially Single-Stranded DNA Donors in Caenorhabditis elegans. Genetics 210, 781-787.

Feng, C., Thyagarajan, P., Shorey, M., Seebold, D.Y., Weiner, A.T., Albertson, R.M., Rao, K.S., Sagasti, A., Goetschius, D.J., and Rolls, M.M. (2019). Patronin-mediated minus end growth is required for dendritic microtubule polarity. J Cell Biol 218, 2309-2328.

Goodwin, S.S., and Vale, R.D. (2010). Patronin regulates the microtubule network by protecting microtubule minus ends. Cell 143, 263-274.

Guedes-Dias, P., and Holzbaur, E.L.F. (2019). Axonal transport: Driving synaptic function. Science 366. 
Guedes-Dias, P., Nirschl, J.J., Abreu, N., Tokito, M.K., Janke, C., Magiera, M.M., and Holzbaur, E.L.F. (2018). Kinesin-3 Responds to Local Microtubule Dynamics to Target Synaptic Cargo Delivery to the Presynapse. Curr. Biol.

Guillabert-Gourgues, A., Jaspard-Vinassa, B., Bats, M.-L., Sewduth, R.N., Franzl, N., Peghaire, C., Jeanningros, S., Moreau, C., Roux, E., Larrieu-Lahargue, F., et al. (2016). Kif26b controls endothelial cell polarity through the Dishevelled/Daam1-dependent planar cell polarity-signaling pathway. Mol. Biol. Cell 27, 941-953.

Hall, D.H., and Hedgecock, E.M. (1991). Kinesin-related gene unc-104 is required for axonal transport of synaptic vesicles in C. elegans. Cell 65, 837-847.

Hao, H.-X., Xie, Y., Zhang, Y., Charlat, O., Oster, E., Avello, M., Lei, H., Mickanin, C., Liu, D., Ruffner, H., et al. (2012). ZNRF3 promotes Wnt receptor turnover in an R-spondin-sensitive manner. Nature 485, 195200.

Harada, A., Oguchi, K., Okabe, S., Kuno, J., Terada, S., Ohshima, T., Sato-Yoshitake, R., Takei, Y., Noda, T., and Hirokawa, N. (1994). Altered microtubule organization in small-calibre axons of mice lacking tau protein. Nature 369, 488-491.

Hata, Y., Butz, S., and Südhof, T.C. (1996). CASK: a novel dlg/PSD95 homolog with an N-terminal calmodulin-dependent protein kinase domain identified by interaction with neurexins. J Neurosci 16, 2488-2494.

He Chun-Wei, Liao Chien-Po, and Pan Chun-Liang Wnt signalling in the development of axon, dendrites and synapses. Open Biology 8, 180116.

Jiang, K., Hua, S., Mohan, R., Grigoriev, I., Yau, K.W., Liu, Q., Katrukha, E.A., Altelaar, A.F.M., Heck, A.J.R., Hoogenraad, C.C., et al. (2014). Microtubule minus-end stabilization by polymerization-driven CAMSAP deposition. Dev Cell 28, 295-309.

Klassen, M.P., and Shen, K. (2007). Wnt signaling positions neuromuscular connectivity by inhibiting synapse formation in C. elegans. Cell 130, 704-716.

Koles, K., and Budnik, V. (2012). Wnt signaling in neuromuscular junction development. Cold Spring Harb Perspect Biol 4.

Koo, B.-K., Spit, M., Jordens, I., Low, T.Y., Stange, D.E., van de Wetering, M., van Es, J.H., Mohammed, S., Heck, A.J.R., Maurice, M.M., et al. (2012). Tumour suppressor RNF43 is a stem-cell E3 ligase that induces endocytosis of Wnt receptors. Nature 488, 665-669.

Koushika, S.P., Schaefer, A.M., Vincent, R., Willis, J.H., Bowerman, B., and Nonet, M.L. (2004). Mutations in Caenorhabditis elegans cytoplasmic dynein components reveal specificity of neuronal retrograde cargo. J Neurosci 24, 3907-3916.

Kreutzberg, G.W. (1969). Neuronal dynamics and axonal flow. IV. Blockage of intra-axonal enzyme transport by colchicine. Proc. Natl. Acad. Sci. U.S.A. 62, 722-728. 
Kurshan, P.T., Merrill, S.A., Dong, Y., Ding, C., Hammarlund, M., Bai, J., Jorgensen, E.M., and Shen, K. (2018). $Y$-Neurexin and Frizzled Mediate Parallel Synapse Assembly Pathways Antagonized by Receptor Endocytosis. Neuron 100, 150-166.e4.

Lüchtenborg, A.-M., Solis, G.P., Egger-Adam, D., Koval, A., Lin, C., Blanchard, M.G., Kellenberger, S., and Katanaev, V.L. (2014). Heterotrimeric Go protein links Wnt-Frizzled signaling with ankyrins to regulate the neuronal microtubule cytoskeleton. Development 141, 3399-3409.

Maeder, C.I., Shen, K., and Hoogenraad, C.C. (2014). Axon and dendritic trafficking. Current Opinion in Neurobiology 27, 165-170.

Marcette, J.D., Chen, J.J., and Nonet, M.L. (2014). The Caenorhabditis elegans microtubule minus-end binding homolog PTRN-1 stabilizes synapses and neurites. ELife 3, e01637.

Meng, L., Chen, C., and Yan, D. (2016). Regulation of Gap Junction Dynamics by UNC-44/ankyrin and UNC-33/CRMP through VAB-8 in C. elegans Neurons. PLoS Genet 12, e1005948.

Meng, W., Mushika, Y., Ichii, T., and Takeichi, M. (2008). Anchorage of microtubule minus ends to adherens junctions regulates epithelial cell-cell contacts. Cell 135, 948-959.

Miech, C., Pauer, H.-U., He, X., and Schwarz, T.L. (2008). Presynaptic local signaling by a canonical wingless pathway regulates development of the Drosophila neuromuscular junction. J Neurosci 28 , 10875-10884.

Mizumoto, K., and Shen, K. (2013). Two Wnts instruct topographic synaptic innervation in C. elegans. Cell Reports 5, 389-396.

Moffat, L.L., Robinson, R.E., Bakoulis, A., and Clark, S.G. (2014). The conserved transmembrane RING finger protein PLR-1 downregulates Wnt signaling by reducing Frizzled, Ror and Ryk cell-surface levels in C. elegans. Development 141, 617-628.

Muhammad, K., Reddy-Alla, S., Driller, J.H., Schreiner, D., Rey, U., Böhme, M.A., Hollmann, C., Ramesh, N., Depner, H., Lützkendorf, J., et al. (2015). Presynaptic spinophilin tunes neurexin signalling to control active zone architecture and function. Nat Commun 6, 8362.

Mukherjee, K., Sharma, M., Urlaub, H., Bourenkov, G.P., Jahn, R., Südhof, T.C., and Wahl, M.C. (2008). CASK Functions as a Mg2+-independent neurexin kinase. Cell 133, 328-339.

Nibbeling, E.A.R., Duarri, A., Verschuuren-Bemelmans, C.C., Fokkens, M.R., Karjalainen, J.M., Smeets, C.J.L.M., de Boer-Bergsma, J.J., van der Vries, G., Dooijes, D., Bampi, G.B., et al. (2017). Exome sequencing and network analysis identifies shared mechanisms underlying spinocerebellar ataxia. Brain 140, 2860-2878.

Niwa, S., Lipton, D.M., Morikawa, M., Zhao, C., Hirokawa, N., Lu, H., and Shen, K. (2016). Autoinhibition of a Neuronal Kinesin UNC-104/KIF1A Regulates the Size and Density of Synapses. Cell Reports 16, 21292141.

Okada, Y., Yamazaki, H., Sekine-Aizawa, Y., and Hirokawa, N. (1995). The neuron-specific kinesin superfamily protein KIF1A is a unique monomeric motor for anterograde axonal transport of synaptic vesicle precursors. Cell 81, 769-780. 
Ou, C.Y., Poon, V.Y., Maeder, C.I., Watanabe, S., Lehrman, E.K., Fu, A.K., Park, M., Fu, W.Y., Jorgensen, E.M., Ip, N.Y., et al. (2010). Two cyclin-dependent kinase pathways are essential for polarized trafficking of presynaptic components. Cell 141, 846-858.

Owald, D., Khorramshahi, O., Gupta, V.K., Banovic, D., Depner, H., Fouquet, W., Wichmann, C., Mertel, S., Eimer, S., Reynolds, E., et al. (2012). Cooperation of Syd-1 with Neurexin synchronizes pre- with postsynaptic assembly. Nat Neurosci 15, 1219-1226.

Pack-Chung, E., Kurshan, P.T., Dickman, D.K., and Schwarz, T.L. (2007). A Drosophila kinesin required for synaptic bouton formation and synaptic vesicle transport. Nat. Neurosci. 10, 980-989.

Pani, A.M., and Goldstein, B. (2018). Direct visualization of a native Wnt in vivo reveals that a long-range Wnt gradient forms by extracellular dispersal. Elife 7.

Qu, X., Kumar, A., Blockus, H., Waites, C., and Bartolini, F. (2019). Activity-Dependent Nucleation of Dynamic Microtubules at Presynaptic Boutons Controls Neurotransmission. Curr Biol 29, 4231-4240.e5.

Richardson, C.E., Spilker, K.A., Cueva, J.G., Perrino, J., Goodman, M.B., and Shen, K. (2014). PTRN-1, a microtubule minus end-binding CAMSAP homolog, promotes microtubule function in Caenorhabditis elegans neurons. ELife 3, e01498.

Rizalar, F.S., Roosen, D.A., and Haucke, V. (2021). A Presynaptic Perspective on Transport and Assembly Mechanisms for Synapse Formation. Neuron 109, 27-41.

Sanes, J.R., and Zipursky, S.L. (2020). Synaptic Specificity, Recognition Molecules, and Assembly of Neural Circuits. Cell 181, 536-556.

Soundararajan, H.C., and Bullock, S.L. (2014). The influence of dynein processivity control, MAPs, and microtubule ends on directional movement of a localising mRNA. ELife 3, e01596.

Stucchi, R., Plucińska, G., Hummel, J.J.A., Zahavi, E.E., Guerra San Juan, I., Klykov, O., Scheltema, R.A., Altelaar, A.F.M., and Hoogenraad, C.C. (2018). Regulation of KIF1A-Driven Dense Core Vesicle Transport: Ca2+/CaM Controls DCV Binding and Liprin- $\alpha /$ TANC2 Recruits DCVs to Postsynaptic Sites. Cell Rep 24, 685-700.

Südhof, T.C. (2017). Synaptic Neurexin Complexes: A Molecular Code for the Logic of Neural Circuits. Cell $171,745-769$.

Südhof, T.C. (2018). Towards an Understanding of Synapse Formation. Neuron 100, 276-293.

Sugie, A., Hakeda-Suzuki, S., Suzuki, E., Silies, M., Shimozono, M., Möhl, C., Suzuki, T., and Tavosanis, G. (2015). Molecular Remodeling of the Presynaptic Active Zone of Drosophila Photoreceptors via ActivityDependent Feedback. Neuron 86, 711-725.

Susman, M.W., Karuna, E.P., Kunz, R.C., Gujral, T.S., Cantú, A.V., Choi, S.S., Jong, B.Y., Okada, K., Scales, M.K., Hum, J., et al. (2017). Kinesin superfamily protein Kif26b links Wnt5a-Ror signaling to the control of cell and tissue behaviors in vertebrates. Elife 6 .

Tan, R., Foster, P.J., Needleman, D.J., and McKenney, R.J. (2018). Cooperative Accumulation of DyneinDynactin at Microtubule Minus-Ends Drives Microtubule Network Reorganization. Dev Cell 44, 233-

247.e4. 
Terabayashi, T., Sakaguchi, M., Shinmyozu, K., Ohshima, T., Johjima, A., Ogura, T., Miki, H., and Nishinakamura, R. (2012). Phosphorylation of Kif26b promotes its polyubiquitination and subsequent proteasomal degradation during kidney development. PLoS ONE 7, e39714.

Vukoja, A., Rey, U., Petzoldt, A.G., Ott, C., Vollweiter, D., Quentin, C., Puchkov, D., Reynolds, E., Lehmann, M., Hohensee, S., et al. (2018). Presynaptic Biogenesis Requires Axonal Transport of Lysosome-Related Vesicles. Neuron 99, 1216-1232.e7.

Wang, L., Tanaka, Y., Wang, D., Morikawa, M., Zhou, R., Homma, N., Miyamoto, Y., and Hirokawa, N. (2018). The Atypical Kinesin KIF26A Facilitates Termination of Nociceptive Responses by Sequestering Focal Adhesion Kinase. Cell Rep 24, 2894-2907.

Wang, S., Wu, D., Quintin, S., Green, R.A., Cheerambathur, D.K., Ochoa, S.D., Desai, A., and Oegema, K. (2015). NOCA-1 functions with gamma-tubulin and in parallel to Patronin to assemble non-centrosomal microtubule arrays in. ELife 4.

White, J.G., Southgate, E., Thomson, J.N., and Brenner, S. (1986). The structure of the nervous system of the nematode Caenorhabditis elegans. Philosophical Transactions of the Royal Society of London. Series B, Biological Sciences 314, 1-340.

Wightman, B., Clark, S.G., Taskar, A.M., Forrester, W.C., Maricq, A.V., Bargmann, C.I., and Garriga, G. (1996). The $C$. elegans gene vab-8 guides posteriorly directed axon outgrowth and cell migration. Development 122, 671-682.

van de Willige, D., Hoogenraad, C.C., and Akhmanova, A. (2016). Microtubule plus-end tracking proteins in neuronal development. Cell Mol Life Sci 73, 2053-2077.

Woehlke, G., Ruby, A.K., Hart, C.L., Ly, B., Hom-Booher, N., and Vale, R.D. (1997). Microtubule Interaction Site of the Kinesin Motor. Cell 90, 207-216.

Wojcik, M.H., Okada, K., Prabhu, S.P., Nowakowski, D.W., Ramsey, K., Balak, C., Rangasamy, S., Brownstein, C.A., Schmitz-Abe, K., Cohen, J.S., et al. (2018). De novo variant in KIF26B is associated with pontocerebellar hypoplasia with infantile spinal muscular atrophy. American Journal of Medical Genetics Part A 176, 2623-2629.

Wolf, F.W., Hung, M.S., Wightman, B., Way, J., and Garriga, G. (1998). vab-8 is a key regulator of posteriorly directed migrations in $\mathrm{C}$. elegans and encodes a novel protein with kinesin motor similarity. Neuron 20,655-666.

Wong, M.Y., Zhou, C., Shakiryanova, D., Lloyd, T.E., Deitcher, D.L., and Levitan, E.S. (2012). Neuropeptide delivery to synapses by long-range vesicle circulation and sporadic capture. Cell 148, 1029-1038.

Wu, Y.E., Huo, L., Maeder, C.I., Feng, W., and Shen, K. (2013). The balance between capture and dissociation of presynaptic proteins controls the spatial distribution of synapses. Neuron 78, 994-1011.

Xuan, Z., Manning, L., Nelson, J., Richmond, J.E., Colón-Ramos, D.A., Shen, K., and Kurshan, P.T. (2017). Clarinet (CLA-1), a novel active zone protein required for synaptic vesicle clustering and release. Elife 6 . Yogev, S., and Shen, K. (2014). Cellular and molecular mechanisms of synaptic specificity. Annu Rev Cell Dev Biol 30, 417-437. 
Yogev, S., Cooper, R., Fetter, R., Horowitz, M., and Shen, K. (2016). Microtubule Organization Determines Axonal Transport Dynamics. Neuron 92, 449-460.

Yogev, S., Maeder, C.I., Cooper, R., Horowitz, M., Hendricks, A.G., and Shen, K. (2017). Local inhibition of microtubule dynamics by dynein is required for neuronal cargo distribution. Nature Communications 8 , 15063.

Zhai, R.G., Vardinon-Friedman, H., Cases-Langhoff, C., Becker, B., Gundelfinger, E.D., Ziv, N.E., and Garner, C.C. (2001). Assembling the presynaptic active zone: a characterization of an active one precursor vesicle. Neuron 29, 131-143.

Zhou, R., Niwa, S., Homma, N., Takei, Y., and Hirokawa, N. (2009). KIF26A is an unconventional kinesin and regulates GDNF-Ret signaling in enteric neuronal development. Cell 139, 802-813. 